\title{
Variational approach in weighted Sobolev spaces to scattering by unbounded rough surfaces
}

Article

Published Version

Chandler-Wilde, S. N. and Elschner, J. (2010) Variational approach in weighted Sobolev spaces to scattering by unbounded rough surfaces. SIAM Journal on Mathematical Analysis (SIMA), 42 (6). pp. 2554-2580. ISSN 0036-1410 doi: https://doi.org/10.1137/090776111 Available at https://centaur.reading.ac.uk/18779/

It is advisable to refer to the publisher's version if you intend to cite from the work. See Guidance on citing.

Published version at: http://dx.doi.org/10.1137/090776111

To link to this article DOI: http://dx.doi.org/10.1137/090776111

Publisher: Society for Industrial and Applied Mathematics

All outputs in CentAUR are protected by Intellectual Property Rights law, including copyright law. Copyright and IPR is retained by the creators or other copyright holders. Terms and conditions for use of this material are defined in the End User Agreement.

www.reading.ac.uk/centaur 
Central Archive at the University of Reading

Reading's research outputs online 


\title{
VARIATIONAL APPROACH IN WEIGHTED SOBOLEV SPACES TO SCATTERING BY UNBOUNDED ROUGH SURFACES*
}

\author{
SIMON N. CHANDLER-WILDE ${ }^{\dagger}$ AND JOHANNES ELSCHNER ${ }^{\ddagger}$
}

\begin{abstract}
We consider the problem of scattering of time harmonic acoustic waves by an unbounded sound soft surface which is assumed to lie within a finite distance of some plane. The paper is concerned with the study of an equivalent variational formulation of this problem set in a scale of weighted Sobolev spaces. We prove well-posedness of this variational formulation in an energy space with weights which extends previous results in the unweighted setting [S. Chandler-Wilde and P. Monk, SIAM J. Math. Anal., 37 (2005), pp. 598-618] to more general inhomogeneous terms in the Helmholtz equation. In particular, in the two-dimensional case, our approach covers the problem of plane wave incidence, whereas in the three-dimensional case, incident spherical and cylindrical waves can be treated. As a further application of our results, we analyze a finite section type approximation, whereby the variational problem posed on an infinite layer is approximated by a variational problem on a bounded region.
\end{abstract}

Key words. nonsmooth boundary, radiation condition, variational formulation, weighted Sobolev spaces, Helmholtz equation

AMS subject classifications. 35J05, 35J20, 35J25, 42B10, 78A45

DOI. $10.1137 / 090776111$

1. Introduction. This paper is concerned with the analysis of problems of scattering by unbounded surfaces, in particular, with what are termed rough surface scattering problems in the engineering literature. By the phrase rough surface, we will denote throughout a surface which is a (usually nonlocal) perturbation of an infinite plane surface such that the surface lies within a finite distance of the original plane. Rough surface scattering problems in this sense arise frequently in applications, for example, in modeling acoustic and electromagnetic wave propagation over outdoor ground and sea surfaces, and have been studied extensively in the physics and engineering literature from the points of view of developing effective numerical algorithms or asymptotic or statistical approximation methods (see, e.g., Ogilvy [33], Voronovich [42], Saillard and Sentenac [35], Warnick and Chew [43], DeSanto [18], and Elfouhaily and Guerin [19]).

Despite this extensive practical interest, relatively little mathematical analysis of these problems has been carried out. In particular, only in the last five years have the first results been obtained, establishing well-posedness for three-dimensional (3D) rough surface scattering problems, using integral equation methods (see ChandlerWilde, Heinemeyer, and Potthast [13,14] and Thomas [39]) or variational formulations (see Chandler-Wilde and Monk [11], Chandler-Wilde, Monk, and Thomas [15], and Thomas [39]). The variational approach proposed in [11] for the sound soft acoustic problem leads to explicit bounds on the solution in terms of the data and applies to a rather general class of nonsmooth unbounded surfaces. The approach in [11] is

* Received by the editors November 5, 2009; accepted for publication (in revised form) August 3, 2010; published electronically October 12, 2010.

http://www.siam.org/journals/sima/42-6/77611.html

$\dagger$ Department of Mathematics, University of Reading, Whiteknights, P.O. Box 220, Berkshire, RG6 6AX, UK (S.N.Chandler-Wilde@reading.ac.uk).

${ }^{\ddagger}$ Weierstrass Institute for Applied Analysis and Stochastics, Mohrenstr. 39, 10117 Berlin, Germany (elschner@wias-berlin.de). 
extended to more general acoustic scattering problems in [39], including problems of scattering by impedance surfaces and by inhomogeneous layers (see [15]).

In this paper we will rigorously analyze time harmonic acoustic scattering, seeking to solve the Helmholtz equation with wave number $k>0$,

$$
\Delta u+k^{2} u=g,
$$

in the perturbed half-plane or half-space $D \subset \mathbb{R}^{n}, n=2,3$. The scattering surface $\Gamma:=\partial D$ is assumed to lie within a finite distance of some plane; for example, it may be the graph of an arbitrary bounded continuous function. Moreover, we assume that the support of $g$ lies in an infinite layer $S_{0}$ of finite thickness between the surface $\Gamma$ and some plane $\Gamma_{0}$ above $\Gamma$ and that $g$ belongs to a weighted $L^{2}$ space $\left(1+r^{2}\right)^{\varrho / 2} L^{2}\left(S_{0}\right)$ for some $|\varrho|<1$, where $r$ denotes the radial distance within the layer $S_{0}$. This function space setting with weights acting only along the boundary of the unbounded domain $D$ covers the important problems of plane wave incidence in the two-dimensional (2D) case and of incident spherical and cylindrical waves in the 3D case; see section 5. While the methods we use and the results we derive can be adapted to other boundary conditions, to keep things specific and to make use of earlier results [11, 15], we will restrict our attention to the simplest case when a homogeneous Dirichlet boundary condition $u=0$ holds on $\Gamma$. The problem formulation is completed by a suitable radiation condition, expressing that the wave scattered by the surface must radiate away from the surface, and the introduced weights allow us to integrate this condition into an equivalent variational formulation of the Dirichlet problem.

This paper is closest in its results to Chandler-Wilde and Monk [11], who studied the same Dirichlet scattering problem in the case of right-hand sides $g \in L^{2}\left(S_{0}\right)$. Extending the approach of [11] in the unweighted space setting, we introduce an equivalent variational formulation of this problem in an energy space with weights which decay or increase as $\left(1+r^{2}\right)^{\varrho / 2},|\varrho|<1$, on the layer $S_{0}$. Moreover, on the upper boundary $\Gamma_{0}$ of $S_{0}$, the solution is required to satisfy a nonlocal boundary condition involving the exact Dirichlet-to-Neumann map $T$. This condition is often used in a formal manner in the rough surface scattering literature (e.g., [18]) so that, above the rough surface $\Gamma$ and the support of $g$, the solution can be represented in integral form as a superposition of upward traveling and evanescent plane waves. This radiation condition is equivalent to the upward propagating radiation condition proposed for 2 D rough surface scattering problems in [10] and has recently been analyzed carefully in the 2D case by Arens and Hohage [4]. Arens and Hohage also propose a further equivalent radiation condition (a "pole condition").

In contrast to the general case of a nonlocally perturbed plane surface, there is already a vast literature on the variational approach applied to periodic diffractive structures (diffraction gratings) or to locally perturbed plane scatterers; see, e.g., Kirsch [27], Bonnet-Bendhia and Starling [6], Elschner and Schmidt [20], Bao and Dobson [5], Elschner et al. [21], Ammari, Bao, and Wood [1], and Elschner and Yamamoto [22]. The assumption made in all of these papers leads to a variational problem over a bounded region so that compact imbedding arguments can be applied and that the sesquilinear form that arises satisfies a Gårding inequality which simplifies the mathematical arguments considerably compared to the cases studied in [11, 15, 39]. However, the detection of eigenfunctions of such periodic diffraction problems (e.g., trapped-mode solutions along diffraction gratings) requires more sophisticated methods, and we refer to Kamotskiǐ and Nazarov [25, 26] and Nazarov [31] for important results in this direction. 
This paper is organized as follows. In sections 2 and 3 we formulate the boundary value problem and its variational formulation precisely, and we give the details about our assumptions on $D$ and about the radiation condition we impose. Section 3 is also devoted to new continuity properties of the DtN map $T$ in weighted Sobolev spaces on $\Gamma_{0}$.

In section 4 we study the well-posedness of the variational formulation in the energy space with weights. Our main result, Theorem 4.1, is to show, for a range of increasing and decreasing weights, that the problem is well-posed in the weighted space setting if and only if it is well-posed in the unweighted space setting. This result depends on technical estimates of the commutator of the DtN map $T$ and the operation of multiplication by the weight function; see Theorem 3.1. Combining this result with previous results on well-posedness in the unweighted setting for sound soft scattering [11], we are able to show well-posedness in a weighted space setting.

In section 5, to illustrate the importance of these results, we make two applications. First, in the 2D case, we prove existence of solution to the problem of plane wave scattering by an unbounded sound soft surface, extending previous results derived for the case when the boundary is the graph of a sufficiently smooth function [17] to much more general surface profiles. We note that, even in the well-studied case when the boundary is periodic (is a diffraction grating), the uniqueness result we obtain is a significant extension of the results known to date [22]. We briefly discuss why our methods do not extend to the case of plane wave incidence in the 3D case (indeed, why this problem may not be well-posed), and we apply our results to prove existence of solution to the 3D problems of scattering of incident spherical and cylindrical waves. In the second application of our results, we analyze the approximation of the variational problem in the infinite layer $S_{0}$ by a variational problem in a bounded region (to which finite element methods can then be applied), with this bounded region coinciding with the original layer $S_{0}$ inside a ball of radius $R$. We prove stability and convergence of this approximation procedure and use our weighted space results to prove error estimates as a function of $R$.

The final section 6 is concerned with the proof of our crucial commutator estimates stated in Theorem 3.1. Note that the DtN map $T$ is a pseudodifferental operator on $\mathbb{R}^{n-1}$ with a nonsmooth symbol so that the standard calculus of pseudifferential operators acting in weighted Sobolev spaces (see, e.g., [34]) is not sufficient to obtain the result.

2. The boundary value problem and radiation conditions. Let $\mathrm{x}=$ $\left(x, x_{n}\right) \in \mathbb{R}^{n}(n=2,3)$ with $x \in \mathbb{R}^{n-1}$, and let $D \subset \mathbb{R}^{n}$ be an unbounded domain such that, for some $b<0$,

$$
U_{0} \subset D \subset U_{b}, \quad U_{b}:=\left\{\mathrm{x}: x_{n}>b\right\} .
$$

For $h \in \mathbb{R}$, let $\Gamma_{h}:=\left\{\mathrm{x}: x_{n}=h\right\}$ and $S_{h}:=D \backslash \overline{U_{h}}$. The variational problem will be posed on the open set $S_{0}$ which lies between the rough surface $\Gamma=\partial D$ and the plane (or line) $\Gamma_{0}$ (cf. Figure 2.1). In addition to (2.1), we will assume that $D$ satisfies the condition that

$$
\mathrm{x} \in D \Rightarrow \mathrm{x}+s e_{n} \in D \text { for all } s>0,
$$

where $e_{n}$ denotes the unit vector in direction $x_{n}$. Condition (2.2) is satisfied if $\Gamma$ is the graph of a continuous function but also allows more general domains. We now introduce weighted $L^{2}$ and Sobolev spaces. For $\varrho \in \mathbb{R}, l \in \mathbb{N}$ and a domain $G \subset \mathbb{R}^{n}$, 


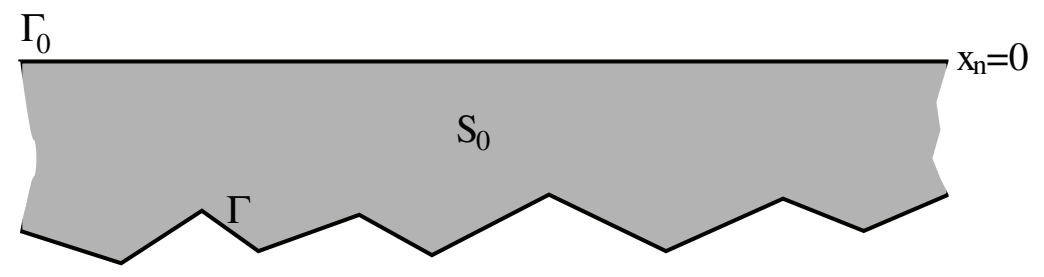

$-x_{n}=b$

FIG. 2.1. Geometrical setting of the scattering problem.

define the Hilbert spaces

$$
\begin{aligned}
& L_{\varrho}^{2}(G):=\left\{u \in L_{\mathrm{loc}}^{2}(G):\left(1+x^{2}\right)^{\varrho / 2} u \in L^{2}(G)\right\}, \\
& H_{\varrho}^{l}(G):=\left\{u \in H_{\mathrm{loc}}^{l}(G):\left(1+x^{2}\right)^{\varrho / 2} u \in H^{l}(G)\right\},
\end{aligned}
$$

equipped with the corresponding canonical norms

$$
\begin{aligned}
\|u\|_{L_{\varrho}^{2}(G)} & =\left(\int_{G}\left|\left(1+x^{2}\right)^{\varrho / 2} u\right|^{2} d \mathrm{x}\right)^{1 / 2}, \\
\|u\|_{H_{\varrho}^{l}(G)} & =\left(\sum_{j=0}^{l} \int_{G}\left|\nabla^{j}\left(1+x^{2}\right)^{\varrho / 2} u\right|^{2} d \mathrm{x}\right)^{1 / 2}, \\
\nabla & =\nabla_{\mathrm{x}}
\end{aligned}
$$

The space $V_{h, \varrho}$ is then defined, for $h \geq 0$, as the closure of $\left\{\left.u\right|_{S_{h}}: u \in C_{0}^{\infty}(D)\right\}$ in the norm

$$
\|u\|_{V_{h, \varrho}}=\|u\|_{H_{\varrho}^{1}\left(S_{h}\right)}=\left(\int_{S_{h}}\left(\left|\left(1+x^{2}\right)^{\varrho / 2} u\right|^{2}+\left|\nabla\left(\left(1+x^{2}\right)^{\varrho / 2} u\right)\right|^{2}\right) d \mathrm{x}\right)^{1 / 2}
$$

We set $V_{0, \varrho}=V_{\varrho}$ in the following, which is the energy space for our variational problem. Moreover, we introduce the weighted Sobolev space

$$
H_{\varrho}^{s}\left(\Gamma_{h}\right):=\left\{v \in H_{\mathrm{loc}}^{s}\left(\Gamma_{h}\right):\left(1+x^{2}\right)^{\varrho / 2} v \in H^{s}\left(\Gamma_{h}\right)\right\}, \quad s, \varrho \in \mathbb{R},
$$

equipped with the canonical norm $\|v\|_{H_{\varrho}^{s}\left(\Gamma_{h}\right)}=\left\|\left(1+x^{2}\right)^{\varrho / 2} v\right\|_{H^{s}\left(\Gamma_{h}\right)}$, where $H^{s}\left(\Gamma_{h}\right)$ is identified with the Sobolev space $H^{s}\left(\mathbb{R}^{n-1}\right)$ with norm

$$
\|v\|_{H^{s}\left(\mathbb{R}^{n-1}\right)}=\left(\int_{\mathbb{R}^{n-1}}\left(1+\xi^{2}\right)^{s}|F v|^{2} d \xi\right)^{1 / 2}
$$

Here $F v$ denotes the Fourier transform of $v$ defined by

$$
F v(\xi)=(2 \pi)^{-(n-1) / 2} \int_{R^{n-1}} \exp (-i x \cdot \xi) v(x) d x, \xi \in \mathbb{R}^{n-1},
$$

with the inverse transform given by

$$
F^{-1} w(x)=(2 \pi)^{-(n-1) / 2} \int_{\mathbb{R}^{n-1}} \exp (i x \cdot \xi) w(\xi) d \xi, x \in \mathbb{R}^{n-1} .
$$

Copyright (c) by SIAM. Unauthorized reproduction of this article is prohibited. 
Note that $F$ is an isometry of $L^{2}\left(\mathbb{R}^{n-1}\right)$ onto itself and also an isometry of $L_{\varrho}^{2}\left(\mathbb{R}^{n-1}\right)$ onto $H^{\varrho}\left(\mathbb{R}^{n-1}\right)$ since, on using the norm (2.4) and the relation $F^{2} u(x)=u(-x), x \in$ $\mathbb{R}^{n-1}$,

$$
\|F u\|_{H^{\varrho}\left(\mathbb{R}^{n-1}\right)}^{2}=\int_{\mathbb{R}^{n-1}}\left(1+x^{2}\right)^{\varrho}\left|F^{2} u\right|^{2} d x=\int_{\mathbb{R}^{n-1}}\left(1+x^{2}\right)^{\varrho}|u|^{2} d x, u \in C_{0}^{\infty}\left(\mathbb{R}^{n-1}\right) .
$$

More generally, $F$ is an isomorphism of $H_{\varrho}^{s}\left(\mathbb{R}^{n-1}\right)$ onto $H_{s}^{\varrho}\left(\mathbb{R}^{n-1}\right)$ for all $s, \varrho \in \mathbb{R}$; see $[34,41]$. From time to time, we will make use of the following lemma, the proof of which is obvious.

Lemma 2.1. Suppose $h \geq 0$ and $\varrho \in \mathbb{R}$. For $u \in V_{h, \varrho}$, let

$$
\|u\|^{\prime}:=\left(\int_{S_{h}}\left(1+x^{2}\right)^{\varrho}\left(|u|^{2}+|\nabla u|^{2}\right) d \mathrm{x}\right)^{1 / 2} .
$$

Then $\|\cdot\|^{\prime}$ is a norm on $V_{h, \varrho}$ that is equivalent to the norm $\|\cdot\|_{V_{h, \varrho}}$; precisely, for some constants $C_{1}$ and $C_{2}$ depending only on $\varrho,\|u\|_{V_{h, \varrho}} \leq C_{1}\|u\|^{\prime} \leq C_{2}\|u\|_{V_{h, \varrho}}$ for all $u \in V_{h, \varrho}$ and all $h \geq 0$.

We now state our boundary value problem, formulated in a weighted space setting. As indicated in the introduction, it is the study of this problem in weighted spaces, and the application of the new results to which this gives rise, which is the object of this paper. Indeed, a main result of this paper will be to show that solvability of this boundary value problem in weighted spaces, for the range of $\varrho$ indicated, is a consequence of its solvability without weights, i.e., for the case $\varrho=0$. (And this is useful since solvability for the simpler case without weights is already established in [11].) After stating the boundary value problem we will comment in the remainder of this section on how the radiation condition is to be understood, in particular, when $\varrho<0$. We will also comment on the restriction on the range of $\varrho(|\varrho|<1)$ in the statement of the boundary value problem, explaining why this range is natural and sharp. Precisely, we will point out that the radiation condition (2.5) does not make sense for all $u \in H_{\varrho}^{1 / 2}$ when $\varrho \leq-1$, and we will show that the boundary value problem is not, in general, solvable for $\varrho \geq 1$.

The boundary value problem $(B V P)$. Given $g \in L_{\varrho}^{2}(D)$, with $|\varrho|<1$ and $\operatorname{supp} g \subset$ $\overline{S_{0}}$, find $u \in H_{l o c}^{1}(D)$ such that $\left.u\right|_{S_{h}} \in V_{h, \varrho}$ for every $h>0$,

$$
\left(\Delta+k^{2}\right) u=g \text { in } D,
$$

in a distributional sense, and the following radiation condition is satisfied:

$$
\begin{aligned}
u(\mathrm{x}) & =F^{-1} \exp \left(-x_{n} \sqrt{\xi^{2}-k^{2}}\right) F u_{0}(\xi) \\
& =(2 \pi)^{-(n-1) / 2} \int_{\mathbb{R}^{n-1}} \exp \left(-x_{n} \sqrt{\xi^{2}-k^{2}}+i x \cdot \xi\right) F u_{0}(\xi) d \xi, \mathrm{x} \in U_{0},
\end{aligned}
$$

where $u_{0}=\left.u\right|_{\Gamma_{0}} \in H_{\varrho}^{1 / 2}\left(\Gamma_{0}\right)$ (from the trace theorem) and $\sqrt{\xi^{2}-k^{2}}=-i \sqrt{k^{2}-\xi^{2}}$ when $|\xi|<k$.

We explain in this paragraph and the next in what sense (2.5) is to be understood and why, in the above formulation, we restrict $\varrho$ to the range $\varrho>-1$ (the restriction to $\varrho<1$ is explained at the end of this section). For $\varrho \geq 0$, the integral (2.5) exists in the ordinary Lebesgue sense since $F u_{0} \in L^{2}\left(\mathbb{R}^{n-1}\right)$. Further, for $u_{0} \in L^{2}\left(\Gamma_{0}\right)$, the radiation condition $(2.5)$ can be written in the alternative form

$$
u(\mathrm{x})=2 \int_{\Gamma_{0}} \frac{\partial \Phi(\mathrm{x}, \mathrm{y})}{\partial y_{n}} u(\mathrm{y}) d s(\mathrm{y})=2 \int_{\mathbb{R}^{n-1}} \frac{\partial \Phi(\mathrm{x}, \mathrm{y})}{\partial y_{n}} u_{0}(y) d y, \mathrm{x} \in U_{0},
$$


where the fundamental solution $\Phi$ of the Helmholtz equation is given by

$$
\Phi(\mathrm{x}, \mathrm{y}):= \begin{cases}\frac{1}{4 \pi} \frac{\exp (i k|\mathrm{x}-\mathrm{y}|)}{|\mathrm{x}-\mathrm{y}|} & \text { if } n=3 \\ \frac{i}{4} H_{0}^{(1)}(k|\mathrm{x}-\mathrm{y}|) & \text { if } n=2\end{cases}
$$

for $\mathrm{x}=\left(x, x_{n}\right), \mathrm{y}=\left(y, y_{n}\right) \in \mathbb{R}^{n}, \mathrm{x} \neq \mathrm{y}$. Here $H_{0}^{(1)}$ is the Hankel function of the first kind of order zero. In the case $n=2,(2.6)$ is just the upward propagating radiation condition (UPRC) proposed in [10], and we refer to [7, Chap. 5.1.1] for $n=3$. For further discussion of the rationale for the radiation condition (2.5) and its relationship to other proposed radiation conditions for rough surface scattering, we refer the reader to [11].

For $\varrho<0$, we understand (2.5) by extending the mapping $u_{0} \in C_{0}^{\infty}\left(\Gamma_{0}\right) \mapsto u(\mathrm{x})$, given by $(2.5)$ for any fixed $\mathrm{x} \in U_{0}$, to a bounded linear functional on $H_{\varrho}^{1 / 2}\left(\Gamma_{0}\right)$. The restriction $\varrho>-1$ arises precisely because this extension is possible only for the range $\varrho>-1$. To see this, we observe that, since $F$ is an isomorphism from $H_{\varrho}^{1 / 2}\left(\mathbb{R}^{n-1}\right)$ to $H_{1 / 2}^{\varrho}\left(\mathbb{R}^{n-1}\right)$, the mapping $u_{0} \mapsto u(\mathrm{x})$ given by $(2.5)$ extends to a bounded linear functional on $H_{\varrho}^{1 / 2}\left(\Gamma_{0}\right)$ for $\varrho<0$ if and only if $f_{\mathrm{x}} \in H_{-1 / 2}^{-\varrho}\left(\mathbb{R}^{n-1}\right)$, where $f_{\mathrm{x}}(\xi):=\exp \left(-x_{n} \sqrt{\xi^{2}-k^{2}}+i x \cdot \xi\right)$. But this holds precisely for $\varrho>-1$; in fact (this can be deduced from (2.6) and Parseval's theorem), the Fourier transform of $f_{\mathrm{x}}$ is given by

$$
F f_{\mathrm{x}}(y)=\left.2(2 \pi)^{(n-1) / 2} \frac{\partial \Phi(\mathrm{x}, \mathrm{y})}{\partial y_{n}}\right|_{y_{n}=0},
$$

and straightforward explicit calculations (see [10] for the case $n=2$ ) yield that

$$
\left|F f_{\mathrm{x}}(y)\right| \sim c_{n} x_{n}|y|^{-(1+n) / 2}
$$

as $|y| \rightarrow \infty$, where the constant $c_{n}$ depends only on $k$ and on the dimension $n$. From this we deduce that $F f_{\mathrm{x}} \in H_{-\varrho}^{-1 / 2}\left(\mathbb{R}^{n-1}\right)$ so that $f_{\mathrm{x}} \in H_{-1 / 2}^{-\varrho}\left(\mathbb{R}^{n-1}\right)$ if and only if $\varrho>-1$. For $\varrho>-1$, the extension of the mapping $u_{0} \mapsto u(\mathrm{x})$, given by (2.5), to a bounded linear functional on $H_{\varrho}^{1 / 2}\left(\Gamma_{0}\right)$ is given explicitly by $(2.6)$, the asymptotics (2.7) guaranteeing the existence of the integral (2.6). Thus (2.6) makes explicit the meaning of $(2.5)$ in the case $-1<\varrho<0$.

Remark 2.2. We note (and this is important in our later applications) that there is a degree of arbitrariness in our radiation conditions (2.5) and (2.6). By this we mean that we could replace $x_{n}$ in (2.5) by $x_{n}-c$ for any $c>0$ (in fact, for any $c \in \mathbb{R}$ such that supp $g \subset \overline{S_{c}}$ and $U_{c} \subset D$ ); the corresponding change to (2.6) would be to replace $\Gamma_{0}$ by $\Gamma_{c}$. We will show in Theorem 4.1 below that the boundary value problem is uniquely solvable. Clearly (by a simple shift in the vertical direction of the axes), we can deduce from this that the above boundary value problem with $x_{n}$ replaced by $x_{n}-c$ in (2.5) is also uniquely solvable. We reassure the reader that these unique solutions are the same! This is demonstrated for the case $\varrho=0$ in [11], and this result, together with the density of $L^{2}\left(S_{0}\right)$ in $L_{\varrho}^{2}\left(S_{0}\right)$ for $\varrho<0$ and the stability results proved in Theorem 4.1, implies that the solutions are the same also for $|\varrho|<1$.

We have explained above the restriction to $\varrho>-1$ in the boundary value problem formulation. We make the restriction $\varrho<1$ because we cannot, in general, expect the 
boundary value problem to be solvable for $\varrho \geq 1$ (with the solution satisfying that $\left.u\right|_{S_{h}} \in V_{h, \varrho}$ for every $h>0$ ). To see this, we consider the instructive case where $D$ is a half-plane or half-space, i.e., $D=U_{c}$, for some $c \in(b, 0)$. Moreover, let us consider the case when $g$ is compactly supported in a ball centered on some point $\mathrm{z} \in S_{0}$ of radius $\epsilon>0$ sufficiently small so that the ball lies in $\overline{S_{0}}$, and further let us assume that $g(\mathrm{x}) \equiv 1$ inside the ball.

We have remarked already that it is shown in [11] that the boundary value problem is uniquely solvable when $\varrho=0$. For the specific case we are considering, the unique solution to this boundary value problem can be written very explicitly. Let $G_{c}(\mathrm{x}, \mathrm{y})$ denote the Dirichlet Green's function for $U_{c}$, given by

$$
G_{c}(\mathrm{x}, \mathrm{y}):=\Phi(\mathrm{x}, \mathrm{y})-\Phi\left(\mathrm{x}, \mathrm{y}_{c}^{\prime}\right)
$$

where $\mathrm{y}_{c}^{\prime}$ denotes the image of the point $\mathrm{y}$ in the plane $y_{n}=c$. Then the solution to the boundary value problem is

$$
u(\mathrm{x})=\left\{\begin{array}{cc}
k^{-2}(1+A f(k|\mathrm{x}-\mathrm{z}|)), & |\mathrm{x}-\mathrm{z}| \leq \epsilon \\
C k^{-2} G_{c}(\mathrm{x}, \mathrm{z}), & |\mathrm{x}-\mathrm{z}|>\epsilon
\end{array}\right.
$$

where the function $f$ is defined by

$$
f(r):= \begin{cases}J_{0}(r), & n=2, \\ \frac{\sin r}{r}, & n=3,\end{cases}
$$

$J_{0}$ is the Bessel function of the first kind of order zero, and the constants $A$ and $C$ are chosen to ensure $C^{1}$ continuity of $u$ across the boundary of the ball; for example, in $3 \mathrm{D}$,

$$
A=Q^{-1}(i \kappa-1) \text { and } C=4 \pi Q^{-1} \exp (-i \kappa)(\kappa \cos (\kappa)-1),
$$

where $Q=\kappa \cos (\kappa)-1+\sin (\kappa)(1-i \kappa)$ and $\kappa=k \epsilon$. It is easy to check that this expression does satisfy $\Delta u+k^{2} u=g$ in $D=U_{c}$ and that $\left.u\right|_{S_{h}} \in V_{h, \varrho}$ for all $h>0$ if $\rho=0$ (indeed, for all $\varrho<1$ ) follows from the asymptotics (2.9) below. To see that $u$, given by (2.8), does satisfy the boundary value problem, it remains to check that $u$ satisfies the radiation condition; to do this, we can show that $u$ satisfies the form (2.6) of the radiation condition by applications of Green's theorem to $G_{0}(\mathrm{x}, \cdot)$ and $u$ in $U_{0}$.

A first observation is that this example demonstrates that, if $g$ is chosen carefully enough, then the above boundary value problem is solvable for all $\varrho \in \mathbb{R}$. For certainly it is true in this example that $g$, being compactly supported, satisfies $g \in L_{\varrho}^{2}(D)$ for all $\varrho \in \mathbb{R}$, and if $\epsilon$ is chosen so that $C=0$, then $u$ is also compactly supported, and so $\left.u\right|_{S_{h}} \in V_{h, \varrho}$, for all $h>0$ and $\varrho \in \mathbb{R}$. But the example, slightly more subtly, also illustrates that, in general, even if $g$ is compactly supported and so satisfies $g \in L_{\varrho}^{2}(D)$ for all $\varrho>0$, we cannot expect that $\left.u\right|_{S_{h}} \in V_{h, \varrho}$ for any $h>0$ and $\varrho \geq 1$ since $\left.u\right|_{S_{0}} \notin V_{0, \varrho}$ for $\varrho=1$ if $C \neq 0$. To see that this is true, it is enough to examine the asymptotics of $G(\mathrm{x}, \mathrm{z})$ as $\mathrm{x} \rightarrow \infty$ in $S_{0}$. From [12, eq. (4.2)] in the 2D case, and by simple direct calculations in the $3 \mathrm{D}$ case we see that (cf. (2.7))

$$
|G(\mathrm{x}, \mathrm{z})| \sim c_{n}^{\prime} k^{4-n}\left(x_{n}-c\right)\left(z_{n}-c\right)(k|\mathrm{x}|)^{-(1+n) / 2} \quad \text { as } \quad|\mathrm{x}| \rightarrow \infty
$$

uniformly in $\mathrm{x} \in S_{0}$ for some constant $c_{n}^{\prime}>0$ depending only on $n$. From these asymptotics, it is an easy calculation to see that $\left.u\right|_{S_{0}} \in L_{\varrho}^{2}\left(S_{0}\right)$ for $\varrho<1$ but not for $\varrho=1$ so that $\left.u\right|_{S_{0}} \notin V_{0,1}$. This example explains why the boundary value problem is not, in general, solvable in the case $\varrho \geq 1$. 
3. The Dirichlet-to-Neumann map and variational formulation. We now consider a variational formulation in weighted Sobolev spaces of the above boundary value problem, which involves the Dirichlet-to-Neumann operator on the artificial boundary $\Gamma_{0}$. As in [11] for $\varrho=0$, there exist continuous trace operators

$$
\gamma_{-}: V_{\varrho} \rightarrow H_{\varrho}^{1 / 2}\left(\Gamma_{0}\right), \gamma_{+}: H_{\varrho}^{1}\left(U_{0} \backslash \overline{U_{h}}\right) \rightarrow H_{\varrho}^{1 / 2}\left(\Gamma_{0}\right), h>0 .
$$

Moreover, if $u_{0} \in C_{0}^{\infty}\left(\Gamma_{0}\right)$ and $u$ is given by (2.5), then

$$
\left.\frac{\partial u}{\partial x_{n}}\right|_{\Gamma_{0}}=-T \gamma_{+} u
$$

where the Dirichlet-to-Neumann map $T$ is given by the pseudodifferential operator

$$
T v(x):=F^{-1} t(\xi) F v(\xi), t(\xi):=\sqrt{\xi^{2}-k^{2}} .
$$

Note that the symbol $t$ of $T$ is not smooth, which makes the study of (3.1) in weighted Sobolev spaces more complicated than in the case $\varrho=0$ (which was treated in [11]). The following commutator estimate is crucial for our analysis, and its proof is carried out in section 6 .

TheOREM 3.1. Consider the commutator

$$
C:=T-\left(a^{2}+x^{2}\right)^{\varrho / 2} T\left(a^{2}+x^{2}\right)^{-\varrho / 2} .
$$

with parameter $a>0$. Then, for $k a \geq 1$ and $|\varrho|<1$, the norm of $C$ on $L^{2}\left(\mathbb{R}^{n-1}\right)$ is bounded by $c(\varrho) \sqrt{k / a}$.

Here and in the following, $c(\varrho)$ denotes a positive constant which depends only on $\varrho$. We remark (cf. the comments at the end of section 2) that the range $|\varrho|<1$ in this theorem is optimal, i.e., this result does not hold for $\varrho= \pm 1$. This follows in part from the duality exhibited between positive and negative values of $\varrho$ in the proof of Theorem 4.1 in section 6 below, which shows that the statement in this theorem holds for $\varrho=-1$ if and only if it holds for $\varrho=1$. Further, if the above theorem were to hold for $\rho=1$, then the proof of Theorem 4.1 below would extend to the case $\rho=1$, which would contradict the example of a solution of the boundary value problem with $g \in L_{1}^{2}(D)$ but $u_{0} \notin V_{0,1}$ at the end of section 2 .

Sometimes the following weaker version of Theorem 3.1 is sufficient, the proof of which is analogous but simpler.

Lemma 3.2. For fixed $k>0$ and $a=1$, the norm of $(3.2)$ on $L^{2}\left(\mathbb{R}^{n-1}\right)$ is bounded by some constant $c(\varrho)$ for $|\varrho|<1$.

The following lemma describes the continuity properties of $T$.

Lemma 3.3. (i) For any $s \in \mathbb{R}, \quad T: H^{s}\left(\mathbb{R}^{n-1}\right) \rightarrow H^{s-1}\left(\mathbb{R}^{n-1}\right)$ is bounded.

(ii) For $|\varrho|<1,0 \leq s \leq 1, \quad T: H_{\varrho}^{s}\left(\mathbb{R}^{n-1}\right) \rightarrow H_{\varrho}^{s-1}\left(\mathbb{R}^{n-1}\right)$ is bounded.

Proof. (i) follows by taking the Fourier transform and using the estimate $|t(\xi)| \leq$ $c\left(1+\xi^{2}\right)^{1 / 2}$ on $\mathbb{R}^{n-1}$; see also [11]. To prove (ii) for $\varrho \neq 0$, we apply the commutator estimate of Lemma 3.2 to get the continuity

$$
\tilde{C}:=\left(1+x^{2}\right)^{-\varrho / 2} T-T\left(1+x^{2}\right)^{-\varrho / 2} \cdot: L^{2}\left(\mathbb{R}^{n-1}\right) \rightarrow L_{\varrho}^{2}\left(\mathbb{R}^{n-1}\right),
$$

and by the continuous imbeddings $H_{\varrho}^{s} \subset L_{\varrho}^{2} \subset H_{\varrho}^{s-1}$, the operator $\tilde{C}: H^{s}\left(\mathbb{R}^{n-1}\right) \rightarrow$ $H_{\varrho}^{s-1}\left(\mathbb{R}^{n-1}\right)$ is bounded. Moreover, by (i), $\left(1+x^{2}\right)^{-\varrho / 2} T: H^{s}\left(\mathbb{R}^{n-1}\right) \rightarrow H_{\varrho}^{s-1}\left(\mathbb{R}^{n-1}\right)$ is bounded so that $T\left(1+x^{2}\right)^{-\varrho / 2} \cdot$ is bounded there, too. This implies the result. 
To state the variational formulation of (BVP), we use the notation

$$
(u, v):=\int_{S_{0}} u \bar{v} d \mathrm{x}
$$

and define the continuous sesquilinear form $B: V_{\varrho} \times V_{-\varrho} \rightarrow \mathbb{C}$ by

$$
B(u, v):=(\nabla u, \nabla v)-k^{2}(u, v)+\int_{\Gamma_{0}} \gamma_{-} \bar{v} T \gamma_{-} u d s(\mathrm{x}) .
$$

Note that this sesquilinear form is well defined and continuous on $V_{\varrho} \times V_{-\varrho}$ for $|\varrho|<1$ as a consequence of Lemma 3.3 with $s=1 / 2$.

The variational formulation $(V)$. Given $g \in L_{\varrho}^{2}\left(S_{0}\right),|\varrho|<1$, find $u \in V_{\varrho}$ such that

$$
B(u, v)=-(g, v) \text { for all } v \in V_{-\varrho} .
$$

As in [11], the equivalence of (BVP) and (V) can be proved using the following weighted version of Lemma 3.2 in that paper.

LEMmA 3.4. Let $|\varrho|<1$.

(i) If (2.5) holds with $u_{0} \in H_{\varrho}^{1 / 2}\left(\Gamma_{0}\right)$, then $u \in H_{\varrho}^{1}\left(U_{0} \backslash \overline{U_{h}}\right)$ for every $h>0$.

(ii) Furthermore, we have $\left(\Delta+k^{2}\right) u=0$ in $U_{0}, \gamma_{+} u=u_{0}$, and

$$
\int_{\Gamma_{0}} \bar{v} T \gamma_{+} u d x+k^{2} \int_{U_{0}} u \bar{v} d \mathrm{x}-\int_{U_{0}} \nabla u \cdot \nabla \bar{v} d \mathrm{x}=0 \quad \text { for all } v \in C_{0}^{\infty}(D) .
$$

As in [11] (for $\varrho=0$ ), assertion (ii) is a consequence of (i). We will prove Lemma 3.4 (i) in section 6 by applying our commutator estimates. Following [11], Lemma 3.4 then implies Lemma 3.5.

Lemma 3.5. If $u$ is a solution of (BVP), then $\left.u\right|_{S_{0}}$ satisfies $(\mathrm{V})$. Conversely, let $w$ be a solution of $(\mathrm{V})$. If we set $u=w$ in $S_{0}$ and define $u$ in $U_{0}$ to be the right-hand side of (2.5) with $u_{0}=\gamma_{-} w$, and extend the definition of $g$ to $D$ by setting $g=0$ in $U_{0}$, then $u$ is a solution of (BVP).

Remark 3.6. We note that the equivalence of (BVP) and (V) stated in Lemma 3.5 holds whenever (2.1) holds. In particular, the proof is not dependent on (2.2). Further, we note that there is no requirement that $\Gamma_{0} \subset \overline{S_{0}}$; it may be the case, for example, that $S_{0}$ is a bounded open set, which need not necessarily be connected. In the case that $\Gamma_{0} \not \subset \overline{S_{0}}$, the action of the trace operator $\gamma_{-}$on $V_{\varrho}$ in (3.3) should be understood by extending the definition of the functions in $V_{\varrho}$ by zero to the whole of the strip $U_{b} \backslash \overline{U_{0}}$ from $S_{0}$, which is their initial domain of definition. This implies that, for $u \in V_{\varrho}, \gamma_{-} u=0$ on $\Gamma_{0} \backslash \overline{S_{0}}$.

4. Existence and uniqueness results in weighted spaces. We shall establish that (BVP) and the equivalent problem (V) are uniquely solvable by using the result of [11, Thm. 4.1] in the nonweighted case and a perturbation argument based on the commutator estimates. By Lemma 3.3 (ii), the form (3.3) generates a continuous linear operator $\mathcal{B}_{\varrho}: V_{\varrho} \rightarrow V_{-\varrho}^{*}$, where $V_{-\varrho}^{*}$ is the dual of $V_{-\varrho}$ (the space of continous antilinear functionals on $\left.V_{-\varrho}\right)$ with respect to the scalar product $(\cdot, \cdot)$ in $L^{2}\left(S_{0}\right)$.

THEOREM 4.1. Under the assumptions (2.1), (2.2), and $|\varrho|<1$, the operator $\mathcal{B}_{\varrho}$ is invertible. In particular, $(\mathrm{V})$ and hence $(\mathrm{BVP})$ have a unique solution for all $g \in L_{\varrho}^{2}\left(S_{0}\right)$.

For $\varrho=0$, this was proved in [11, Thm. 4.1] using a Rellich identity and the generalized Lax-Milgram theorem. Moreover, an explicit bound for the norm of $\mathcal{B}_{0}^{-1}$ 
in terms of $k$ and $|b|$ was given there (using wave number dependent Sobolev norms on $S_{0}$ and $\Gamma_{0}$; see Remark 4.2 below). A significant idea in the proof of this theorem for the case $\varrho \neq 0$ is the use of a perturbation argument, reducing the proof of invertibility for $\varrho \neq 0$ to that for $\varrho=0$. This idea has been used previously to study rough surface scattering in $2 \mathrm{D}(n=2)$ in weighted spaces of continuous functions via integral equation methods in $[2,3]$. A commutator result for boundary integral operators (cf. Theorem 4.1) plays in [2] an important role, but the idea there is to prove that the commutator is compact, or at least preserves Fredholmness, rather than to show the stronger and more constructive result that the commutator is sufficiently small in norm. (And it should be noted that the proof of properties of the commutator in [2] is very much more straightforward than the proof of Theorem 3.1, not least because the kernels of the boundary integral operators in [2] are absolutely integrable.) A key ingredient in our commutator estimate, Theorem 3.1, is the parameter $a$ in the weight factor. We note that the idea of introducing such a parameter into commutator estimates goes back at least to Shubin [36, Thm. 5.3], although we seem to be the first to use this idea in an estimate of the commutator of a convolution operator with multiplication by a weight function in the case when the convolution operator has a nonsmooth symbol.

Proof for $\varrho \neq 0$. Introduce equivalent norms $\|u\|_{L_{\varrho}^{2}}=\left\|\left(a^{2}+x^{2}\right)^{\varrho / 2} u\right\|_{L^{2}}$ with parameter $a>0$, and modify the norm (2.3) in $V_{\varrho}$ correspondingly. We will choose $a>0$ sufficiently large, and set, for $u \in V_{\varrho}, \varphi \in V_{-\varrho}$,

$$
v=\left(a^{2}+x^{2}\right)^{\varrho / 2} u \in V_{0}, \quad \psi=\left(a^{2}+x^{2}\right)^{-\varrho / 2} \varphi \in V_{0} .
$$

Then we obtain from (3.3)

$$
B(u, \varphi)=B(v, \psi)+K(v, \psi),
$$

where $K=K_{1}+K_{2}$ with

$$
\begin{aligned}
K_{1}(v, \psi)= & \left(\nabla\left(a^{2}+x^{2}\right)^{-\varrho / 2} v, \nabla\left(a^{2}+x^{2}\right)^{\varrho / 2} \psi\right)-(\nabla v, \nabla \psi) \\
(4.2)= & \left(v \nabla\left(a^{2}+x^{2}\right)^{-\varrho / 2}, \psi \nabla\left(a^{2}+x^{2}\right)^{\varrho / 2}\right)+\left(\nabla v, \psi\left(a^{2}+x^{2}\right)^{-\varrho / 2} \nabla\left(a^{2}+x^{2}\right)^{\varrho / 2}\right) \\
& +\left(v\left(a^{2}+x^{2}\right)^{\varrho / 2} \nabla\left(a^{2}+x^{2}\right)^{-\varrho / 2}, \nabla \psi\right)
\end{aligned}
$$

and

$$
K_{2}(v, \psi)=\int_{\Gamma_{0}}\left\{\left(a^{2}+x^{2}\right)^{\varrho / 2} \bar{\psi} T\left(a^{2}+x^{2}\right)^{-\varrho / 2} v-\bar{\psi} T v\right\} d x=-\int_{\Gamma_{0}} \bar{\psi} C v d x
$$

with $C$ defined in (3.2). For (4.2) we obtain the estimate

$$
\begin{aligned}
\left|K_{1}(v, \psi)\right| \leq & \left(\frac{|\varrho|}{2 a}\right)^{2}\|v\|_{L^{2}\left(S_{0}\right)}\|\psi\|_{L^{2}\left(S_{0}\right)}+\left(\frac{|\varrho|}{2 a}\right)\left(\|\nabla v\|_{L^{2}\left(S_{0}\right)}\|\psi\|_{L^{2}\left(S_{0}\right)}\right. \\
& \left.+\|v\|_{L^{2}\left(S_{0}\right)}\|\nabla \psi\|_{L^{2}\left(S_{0}\right)}\right) \\
\leq & \frac{|\varrho|}{2 a} \max \left(1, \frac{|\varrho|}{2 a}\right)\|v\|_{V_{0}}\|\psi\|_{V_{0}} .
\end{aligned}
$$

Note that

$$
\sup _{S_{0}}\left|\nabla\left(a^{2}+x^{2}\right)^{|\varrho| / 2}\right|\left(a^{2}+x^{2}\right)^{-|\varrho| / 2} \leq|\varrho| / 2 a,
$$

and compare [15, sect. 4$]$. 
Applying Theorem 3.1 to (4.3), we get

$$
\left|K_{2}(v, \psi)\right| \leq c(\varrho) \sqrt{k / a}\left\|\gamma_{-} v\right\|_{L^{2}\left(\Gamma_{0}\right)}\left\|\gamma_{-} \psi\right\|_{L^{2}\left(\Gamma_{0}\right)},
$$

and since $\left\|\gamma_{-} v\right\|_{L^{2}\left(\Gamma_{0}\right)} \leq\left\|\gamma_{-} v\right\|_{H^{1 / 2}\left(\Gamma_{0}\right)} \leq c\|v\|_{V_{0}}$, (4.4) and (4.5) then imply that the norm of the operator $\mathcal{K}_{0}: V_{0} \rightarrow V_{0}^{*}$ generated by the form $K$ tends to zero as $a \rightarrow \infty$. Finally, from (4.1), we have

$$
\mathcal{B}_{\varrho}=\left(a^{2}+x^{2}\right)^{-\varrho / 2}\left(\mathcal{B}_{0}+\mathcal{K}_{0}\right)\left(a^{2}+x^{2}\right)^{\varrho / 2} \cdot .
$$

Since $\mathcal{B}_{0}$ is invertible, this operator is invertible provided that $a$ is sufficiently large.

Remark 4.2. Introducing norms dependent on the wave number $k$ and/or the parameter $a>0$, defined by

$$
\begin{aligned}
|v|_{H^{1 / 2}\left(\Gamma_{0}\right)} & =\left(\int_{\mathbb{R}^{n-1}}\left(k^{2}+\xi^{2}\right)|F v|^{2} d \xi\right)^{1 / 2} \\
|u|_{V_{\varrho}} & =\left(\int_{S_{0}}\left(k^{2}\left|\left(a^{2}+x^{2}\right)^{\varrho / 2} u\right|^{2}+\left|\nabla\left(\left(a^{2}+x^{2}\right)^{\varrho / 2} u\right)\right|^{2}\right) d \mathrm{x}\right)^{1 / 2} \\
|g|_{L_{\varrho}^{2}\left(S_{h}\right)} & =\left(\int_{S_{h}}\left|\left(a^{2}+x^{2}\right)^{\varrho / 2} g\right|^{2} d \mathrm{x}\right)^{1 / 2}
\end{aligned}
$$

on $H^{1 / 2}\left(\Gamma_{0}\right), V_{\varrho}$, and $L_{\varrho}^{2}\left(S_{h}\right)$, respectively, we can obtain a bound of the norm $\left\|\mathcal{B}_{\varrho}^{-1}\right\|$ of $\mathcal{B}_{\varrho}^{-1}: V_{-\varrho}^{*} \rightarrow V_{\varrho}$ in terms of $\kappa=k|b|$, provided that the parameter $a>0$ is chosen sufficiently large. From (4.4) we see that

$$
\left|K_{1}(v, \psi)\right| \leq \frac{|\varrho|}{2 k a}\left(1+\frac{|\varrho|}{2 k a}\right)|v|_{V_{0}}|\psi|_{V_{0}},
$$

and since (cf. [11])

$$
\left\|\gamma_{-} v\right\|_{L^{2}\left(\Gamma_{0}\right)} \leq k^{-1 / 2}\left|\gamma_{-} v\right|_{H^{1 / 2}\left(\Gamma_{0}\right)} \leq k^{-1 / 2}|v|_{V_{0}},
$$

(4.5) implies that

$$
\left|K_{2}(v, \psi)\right| \leq \frac{c(\varrho)}{\sqrt{k a}}|v|_{V_{0}}|\psi|_{V_{0}} .
$$

Thus we have, for $k a \geq 1$ and $|\varrho|<1$,

$$
\left|K_{0}(v, \psi)\right| \leq\left(\frac{|\varrho|}{2 k a}\left(1+\frac{|\varrho|}{2 k a}\right)+\frac{c(\varrho)}{\sqrt{k a}}\right)|v|_{V_{0}}|\psi|_{V_{0}} \leq \frac{|\varrho|+c(\varrho)}{\sqrt{k a}}|v|_{V_{0}}|\psi|_{V_{0}},
$$

so that $\left\|\mathcal{K}_{0}\right\| \leq(|\varrho|+c(\varrho)) / \sqrt{k a}$. Taking the bound

$$
\left\|\mathcal{B}_{0}^{-1}\right\| \leq \gamma:=1+\sqrt{2} \kappa(\kappa+1)^{2}
$$

from [11, Thm. 4.1] and using (4.6), we obtain the norm estimate

$$
\left\|\mathcal{B}_{\varrho}^{-1}\right\| \leq 2 \gamma
$$

Copyright $@$ by SIAM. Unauthorized reproduction of this article is prohibited. 
provided that

$$
\left\|\mathcal{K}_{0}\right\| \leq(|\varrho|+c(\varrho)) / \sqrt{k a} \leq \frac{1}{2 \gamma} \leq \frac{1}{2}\left\|\mathcal{B}_{0}^{-1}\right\|
$$

which holds for $a \geq 4 \gamma^{2}(|\varrho|+c(\varrho))^{2} / k$. Since $(\mathrm{V})$ written in operator form is the equation $\mathcal{B}_{\varrho} u=\tilde{g}$, where $\tilde{g} \in V_{-\varrho}^{*}$ is defined by $\tilde{g}(v)=(g, v), v \in V_{-\varrho}$, this implies that the solution $u$ of $(\mathrm{V})$ satisfies

$$
|u|_{V_{\varrho}} \leq 2 \gamma|\tilde{g}|_{V_{-\varrho}^{*}} \leq 2 \gamma k^{-1}|g|_{L_{\varrho}^{2}\left(S_{0}\right)},
$$

provided $k a \geq \max \left(1,4 \gamma^{2}(|\varrho|+c(\varrho))^{2}\right)$.

\section{Applications.}

5.1. Plane wave incidence, diffraction gratings, and other scattering problems. As an application of Theorem 4.1, the problem of plane wave incidence in the $2 \mathrm{D}$ case $(n=2)$ can be treated. That is, it can be shown, in appropriate function spaces, that the scattering problem for plane wave incidence has exactly one solution in 2D. (For a brief discussion of what goes wrong in the 3D case, see Remark 5.5 below, and see Remark 5.6 for details of 3D scattering problems which can be tackled by Theorem 4.1.) The incident plane wave has the form

$$
v^{i n}(\mathrm{x})=\exp \left(i k\left[\sin \theta x_{1}-\cos \theta x_{2}\right]\right),
$$

where $\theta$ is the angle of incidence, with $|\theta|<\pi / 2$. In this problem, we look for the total field $v=v^{s c}+v^{i n}, v^{s c}$ being the unknown scattered field, such that

$$
\left(\Delta+k^{2}\right) v=0 \text { in } D, \quad v=0 \text { on } \Gamma,
$$

and $v^{s c}$ satisfies an appropriate radiation condition.

This $2 \mathrm{D}$ rough surface scattering problem with plane wave incidence has been treated before, by integral equation methods, in [17], where it is shown that there exists exactly one solution $v \in C^{2}(D) \cap C(\bar{D})$ such that $v$ is bounded in $S_{h}$, for every $h>0$, and $v^{s c}$ satisfies the radiation condition in the form (2.6) (the UPRC in [17]). However, the proof in [17] is only for the case where $\partial D$ is the graph of a sufficiently smooth $\left(C^{1,1}\right)$ function. (This, or at least a restriction to Lyapunov surfaces, is an essential restriction due to the compactness arguments in the existence proofs in [17].) In this section we will establish unique existence of solution for much more general surfaces, with only the constraints (2.1) and (2.2) on $\partial D$ that we impose throughout the paper.

To use the results of the previous section which are formulated in a Sobolev space setting, including the results of Remark 4.2 which are formulated in terms of wave number dependent norms, we will replace the assumption that $v$ is bounded in $S_{h}$ by an assumption that $\left.v\right|_{S_{h}} \in V_{h}^{\infty}$, for all $h>0$, where

$$
V_{h}^{\infty}:=\left\{w \in V_{h,-1}:|w|_{V_{h}^{\infty}}<\infty\right\} .
$$

In this definition the (wave number dependent) norm $|w|_{V_{h}^{\infty}}$ on $V_{h}^{\infty}$ is defined by

$$
|w|_{V_{h}^{\infty}}:=\left.\sup _{m \in \mathbb{Z}}|w|_{S_{h}^{m}}\right|_{H^{1}\left(S_{h}^{m}\right)}, \quad S_{h}^{m}:=\left\{\mathrm{x}=\left(x_{1}, x_{2}\right) \in S_{h}: A m<x_{1}<A(m+1)\right\},
$$


$A>0$ is a parameter at our disposal, and $|\cdot|_{H^{1}\left(S_{h}^{m}\right)}($ cf. Remark 4.2) is a wave number dependent norm on $H^{1}\left(S_{h}^{m}\right)$, equivalent to the usual norm, defined by

$$
|w|_{H^{1}\left(S_{h}^{m}\right)}:=\left(\int_{S_{h}^{m}}\left(k^{2}|w|^{2}+|\nabla w|^{2}\right) d \mathrm{x}\right)^{1 / 2} .
$$

It is easy to see that $V_{h}^{\infty}$ is a Banach space and that the different choices of $A>0$ in the definition of $|\cdot| V_{h}^{\infty}$ provide a family of equivalent norms on $V_{h}^{\infty}$.

In terms of $V_{h}^{\infty}$ our formulation of the plane wave scattering problem is as follows.

Plane wave scattering problem $(P W)$. Given $k>0$ and $\theta \in(-\pi / 2, \pi / 2)$, find $v \in H_{\text {loc }}^{1}(D) \cap C^{2}(D)$ such that $\left.v\right|_{S_{h}} \in V_{h}^{\infty}$, for every $h>0$,

$$
\Delta v+k^{2} v=0 \quad \text { in } D
$$

and such that $v^{s c}:=v-v^{i n}$ satisfies the UPRC (2.6).

Our main result in this subsection is the following, which is an immediate consequence of Theorem 5.3 below.

THEOREM 5.1. The problem $(P W)$ has exactly one solution. Moreover, for every $h>0$, there exists a constant $C_{p}>0$, depending only on $\kappa=k|b|, k h$, and $k A$, such that $\left.|v|_{S_{h}}\right|_{V_{h}^{\infty}} \leq C_{p}$.

An interesting application of this result is the much studied diffraction grating case, where $D$ is periodic in the horizontal direction with some period $A>0$, i.e.,

$$
\mathrm{x}=\left(x_{1}, x_{2}\right) \in D \Leftrightarrow\left(x_{1}+A, x_{2}\right) \in D .
$$

The standard formulation of the problem of plane wave scattering in the diffraction grating case is the following (e.g., [20,22]). In this formulation the function space $V_{h}^{q p, \theta}$ denotes the set of functions in $H_{\mathrm{loc}}^{1}\left(S_{h}\right)$ that vanish on $\partial D$ and are quasiperiodic in the $x_{1}$-direction with the same period and phase shift as the incident wave; more precisely,

$$
V_{h}^{q p, \theta}:=\left\{w \in V_{h}^{\infty}: w\left(\left(x_{1}+A, x_{2}\right)\right)=w(\mathrm{x}) \exp (i k A \sin \theta), \mathrm{x} \in S_{h}\right\} .
$$

Note that, for $w \in V_{h}^{q p, \theta}$, the norm $|w|_{V_{h}^{\infty}}$ simplifies to

$$
|w|_{V_{h}^{\infty}}=\left.|w|_{S_{h}^{0}}\right|_{H^{1}\left(S_{h}^{0}\right)} .
$$

Diffraction grating plane wave scattering problem (DGPW) (e.g., [22]). Given $k>0$ and $\theta \in(-\pi / 2, \pi / 2)$, find $v \in H_{\mathrm{loc}}^{1}(D) \cap C^{2}(D)$ such that $\left.v\right|_{S_{h}} \in V_{h}^{q p, \theta}$, for every $h>0$,

$$
\Delta v+k^{2} v=0 \quad \text { in } D
$$

and such that $v^{s c}:=v-v^{i n}$ satisfies the Rayleigh expansion radiation condition, that is,

$$
v^{s c}(\mathrm{x})=\sum_{m \in \mathbb{Z}} u_{m} \exp \left(i k\left[\alpha_{m} x_{1}+\beta_{m} x_{2}\right]\right), \quad \mathrm{x} \in U_{0},
$$

where the $u_{m}$ are complex constants, $\alpha_{m}:=\sin \theta+2 \pi m /(k A)$, and

$$
\beta_{m}:=\left\{\begin{array}{cc}
\sqrt{1-\alpha_{m}^{2}}, & \left|\alpha_{m}\right| \leq 1, \\
i \sqrt{\alpha_{m}^{2}-1}, & \left|\alpha_{m}\right|>1 .
\end{array}\right.
$$

Copyright (C) by SIAM. Unauthorized reproduction of this article is prohibited. 
It is shown in [22] that (DGPW) has exactly one solution in the case that $\partial D$ is the graph of an (A-periodic) Lipschitz function, by extending well-known arguments (see, e.g., [27]), which apply in the case when $\partial D$ is the graph of a smooth function, to the nonsmooth Lipschitz case. The following corollary of Theorem 5.1 extends that result further to the much more general case, where $\partial D$ is required only to satisfy (2.1), (2.2), and (5.2).

Corollary 5.2. Suppose that (5.2) holds. Then (DGPW) has exactly one solution, and this is the unique solution of $(P W)$.

Proof. Suppose that $v$ satisfies (DGPW). Then it is clear that $v$ satisfies (PW), provided we can show that $v$ satisfying the Rayleigh expansion radiation condition implies that $v$ satisfies the UPRC (2.6). But this is shown in [8]. Conversely, suppose that $v$ satisfies $(\mathrm{PW})$. Then

$$
v\left(\left(x_{1}+A, x_{2}\right)\right)=v(\mathrm{x}) \exp (i k A \sin \theta), \quad \mathrm{x} \in D,
$$

for otherwise $w$, defined by $w(\mathrm{x})=v\left(\left(x_{1}+A, x_{2}\right)\right)$, is another, distinct solution of (PW), which contradicts Theorem 5.1. Thus $v$ satisfies (DGPW) provided that (5.3) holds. But, in the case that (5.4) is satisfied, it is shown in [8] that (2.6) can be written in the form (5.3).

Thus $v$ satisfies (PW) if and only if $v$ satisfies (DGPW), and the corollary follows from Theorem 5.1.

We will prove Theorem 5.1 by applications of Theorem 4.1 and the observations in Remark 4.2. To apply these results, we introduce the function $u \in H_{\mathrm{loc}}^{1}(D) \cap C^{1}(D)$, related to the solution $v$ of $(\mathrm{PW})$ by the formula

$$
u(\mathrm{x}):=v^{i n}(\mathrm{x}) \chi\left(x_{2}\right)+v^{s c}(\mathrm{x})=v(\mathrm{x})+\left(\chi\left(x_{2}\right)-1\right) v^{i n}(\mathrm{x}), \quad \mathrm{x} \in D,
$$

where $\chi \in C^{1}(\mathbb{R})$ is defined by

$$
\chi(t):=\left\{\begin{array}{cc}
1, & t<|b|, \\
\cos ^{2}\left(\frac{\pi(t-|b|)}{2|b|}\right), & |b| \leq t \leq 2|b|, \\
0, & t>|b| .
\end{array}\right.
$$

Moreover, define $g_{P} \in L^{\infty}(D)$ by

$$
g_{P}(\mathrm{x})=\left(\chi^{\prime \prime}\left(x_{2}\right)-2 i k \cos \theta \chi^{\prime}\left(x_{2}\right)\right) v^{i n}(\mathrm{x}), \quad \mathrm{x} \in D,
$$

so that $\left(\Delta+k^{2}\right) u=g_{P}$ in a distributional sense in $D, \operatorname{supp} g_{P} \subset S_{-b}$, and $g_{P} \in L_{\varrho}^{2}(D)$ for $\varrho<-1 / 2$. Then Theorem 5.1 is an immediate consequence of the following result whose proof is largely applications of Theorem 4.1.

Theorem 5.3. Define $g_{P} \in L^{\infty}(D)$ by (5.6). Then $v$ satisfies $(P W)$ if and only if $v$ and $u$ are related by (5.5) and $u$ satisfies the following boundary value problem: given $\varrho \in(-1,-1 / 2)$, find $u \in H_{\text {loc }}^{1}(D)$ such that $\left.u\right|_{S_{h}} \in V_{h, \varrho}$, for every $h>0$,

$$
\left(\Delta+k^{2}\right) u=g_{P} \quad \text { in } \quad D,
$$

in a distributional sense, and $u$ satisfies the radiation condition (2.5) with $x_{n}\left(=x_{2}\right)$ replaced by $x_{2}-b$. Moreover, for every $h>0$, there exists a constant $C_{p}^{\prime}>0$, depending only on $\kappa=k|b|, k h$, and $k A$, such that $\left.|u|_{S_{h}}\right|_{V_{h}^{\infty}} \leq C_{p}^{\prime}$.

Proof. It follows from the observations immediately above the theorem that if $v$ satisfies (PW), then $u$, defined by (5.5), satisfies the above boundary value problem. 
The only difficulty is to show the radiation condition. To see this we note that $v^{s c}$ satisfies the radiation condition (2.6), from which it follows (see [9] and cf. Remark 2.2) that $v^{s c}$ satisfies (2.6) with $\Gamma_{0}$ replaced with $\Gamma_{c}$, for all $c>0$, in particular, with $c=-b$. Since $u=v^{s c}$ in $U_{c}$, it is immediate that $v$ satisfies (2.6) with $\Gamma_{0}$ replaced by $\Gamma_{-b}$, which is equivalent (see Remark 2.2) to (2.5) with $x_{2}$ replaced by $x_{2}+b$.

We next observe that it follows from Theorem 4.1 that the boundary value problem for $u$ has exactly one solution ( $u$ satisfies exactly a boundary value problem of the form of section 2 after vertical translation of the axes by a distance $|b|$ ). The theorem is thus proved if we can show that this solution satisfies that $\left.u\right|_{S_{h}} \in V_{h}^{\infty}$, for every $h>0$, and the bound $\left.|u|_{S_{h}}\right|_{V_{h}^{\infty}} \leq C_{p}^{\prime}$.

To see this we make the following construction. Given $h>0$, set $\tilde{h}:=\max (|b|, h)$ and, for $j \in \mathbb{Z}$, define $D_{j}, g_{j} \in L^{\infty}\left(D_{n}\right)$ and $u_{j} \in H_{\text {loc }}^{1}\left(D_{j}\right)$ by

$$
\begin{gathered}
D_{j}:=\left\{\left(x_{1}+j A, x_{2}-\tilde{h}\right): \mathrm{x}=\left(x_{1}, x_{2}\right) \in D\right\}, \\
g_{j}(\mathrm{x}):=g_{P}\left(\left(x_{1}-j A, x_{2}+\tilde{h}\right)\right), \quad u_{j}(\mathrm{x}):=u\left(\left(x_{1}-j A, x_{2}+\tilde{h}\right)\right), \quad \mathrm{x} \in D_{j} .
\end{gathered}
$$

Then $u_{j}$ satisfies (BVP), with $D$ replaced by $D_{j}$ and $g$ replaced by $g_{j}$ in (BVP). (Since $u$ satisfies (2.5) with $x_{2}$ replaced by $x_{2}-b$, it follows in the first instance that $u_{j}$ satisfies (2.5) with $x_{2}$ replaced with $x_{2}-b-\tilde{h}$, but this implies that (2.5) holds as written, by Remark 2.2.) Thus Theorem 4.1 and Remark 4.2 apply for $\varrho \in(-1,-1 / 2)$. In particular, choosing $k a$ sufficiently large (by Remark 4.2, how large is sufficient depends on only the values of $\kappa$ and $\varrho$ ), it follows from (4.8) that, for some constant $c_{\kappa}>0$ depending only on $\tilde{\kappa}:=k(|b|+\tilde{h})$ and $\varrho,\left|u_{j}\right|_{V_{\varrho}^{j}} \leq c_{\kappa} k^{-1}\left|g_{j}\right|_{L_{\varrho}^{2}\left(S_{0, j}\right)}$, for $j \in \mathbb{Z}$, where $S_{0, j}$ and $V_{\varrho}^{j}$ denote $S_{0}$ and $V_{\varrho}$, respectively, in the case $D=D_{j}$. Since, for some constant $c>0$ depending only on $\varrho$,

$$
\left|g_{j}\right|_{L_{\varrho}^{2}\left(S_{0, j}\right)} \leq\left(\int_{S_{0, j}}\left(a^{2}+x_{1}^{2}\right)^{\varrho} d \mathrm{x}\right)^{1 / 2}\left\|g_{j}\right\|_{L^{\infty}\left(S_{0, j}\right)} \leq c|b|^{1 / 2} a^{\varrho+1 / 2}\left\|g_{P}\right\|_{L^{\infty}\left(S_{-b}\right)},
$$

we see that

$$
\left|u_{j}\right|_{V_{\varrho}^{j}} \leq c_{\kappa}^{\prime}|b|^{-1 / 2} a^{\varrho+1 / 2}, \quad j \in \mathbb{Z},
$$

for some constant $c_{\kappa}^{\prime}$ depending only on $\tilde{\kappa}$ and $\varrho$. Careful calculations yield that, again for some constant $c>0$ depending only on $\varrho$ and where $S_{0, j}^{0}$ denotes $S_{0}^{0}$ in the case $D=D_{j}$

$$
\left.\left|u_{j}\right|_{S_{0, j}^{0}}\right|_{H^{1}\left(S_{0, j}^{0}\right)} \leq c\left(a^{2}+A^{2}\right)^{-\varrho / 2}\left|u_{j}\right|_{V_{\varrho}^{j}}, \quad j \in \mathbb{Z} .
$$

Thus

$$
\left.|u|_{S_{h}}\right|_{V_{h}^{\infty}} \leq\left.|u|_{S_{\tilde{h}}}\right|_{V_{\tilde{h}}^{\infty}}=\left.\sup _{j \in \mathbb{Z}}\left|u_{j}\right|_{S_{0, j}^{0}}\right|_{H^{1}\left(S_{0, j}^{0}\right)} \leq c_{\kappa}^{\prime \prime}\left(1+A^{2} / a^{2}\right)^{|\varrho| / 2}(k a)^{1 / 2},
$$

where $c_{\kappa}^{\prime \prime}>0$ depends only on $\tilde{\kappa}$ and $\varrho$. Now this bound holds for all $k a$ sufficiently large, but how large is sufficient depends only on $\tilde{\kappa}$ and $\varrho$. Thus, choosing such a sufficiently large $k a$ and choosing, say, $\varrho=-3 / 4$, we see that the theorem is proved.

Remark 5.4. Theorem 5.3 explains how, in the $2 \mathrm{D}$ case, (PW) is equivalent to an instance of (BVP). This enables us to prove solvability of (PW) via the reformulation of (BVP) in variational form as (V). This is convenient for the purpose 
of proving Theorem 5.1 above, but, as a starting point for numerical computation (cf. section 5.2 below), we would choose rather to reformulate (PW) directly in variational form. Arguing analogously to the proof of Lemma 3.5, in particular, using Lemma 3.4 which applies to $v^{s c}$, we can show that $v$ satisfies (PW) if and only if, for some $\varrho \in(-1,-1 / 2),\left.v\right|_{S_{0}}$ satisfies the following variational problem: find $v \in V_{\varrho}$ such that

$$
B(u, w)=G(w) \quad \text { for all } w \in V_{-\varrho},
$$

where $G \in V_{-\varrho}^{*}$ is defined by

$$
G(w)=\int_{\Gamma_{0}} \gamma_{-} \bar{w}\left(\frac{\partial v^{i n}}{\partial x_{2}}+T \gamma_{-} v^{i n}\right) d s(x), \quad w \in V_{-\varrho} .
$$

The restriction to the range $\varrho<-1 / 2$ arises since $v^{\text {in }} \in V_{\varrho}$ for $\varrho<-1 / 2$ but not for $\varrho=1 / 2$. Having solved this variational problem to determine $\left.v\right|_{S_{0}}, v$ is determined throughout $D$ through (2.6) satisfied by $v^{s c}$. Of course, this variational formulation is well-posed by Theorem 4.1.

Remark 5.5. The above results show that the problem of plane wave incidence is well-posed in the $2 \mathrm{D}$ case. In the $3 \mathrm{D}$ case, it seems to us likely that a solution to the problem of plane wave incidence does not exist for every choice of domain $D$ satisfying (2.1) and (2.2). Certainly the methods of argument above do not extend to the 3D case, for, in the 3D case, $g_{P}$ in Theorem 5.3 is in $L_{\varrho}^{2}(D)$ only for $\varrho<-1$ and $G$ given by (5.8) is in $V_{-\varrho}^{*}$ only for $\varrho<-1$, so that Theorem 4.1 does not apply. Further, even the formulation of the 3D plane wave problem appears problematic in 3D. Precisely, just as the radiation condition (2.5) does not extend to a bounded linear functional on $H_{\varrho}^{1 / 2}\left(\Gamma_{0}\right)$ for $\varrho<-1$, it does not extend to a bounded linear functional on $L^{\infty}\left(\Gamma_{0}\right)$ (which would require that the integral in (2.6) be absolutely convergent for every $u_{0} \in L^{\infty}\left(\Gamma_{0}\right)$, which is true in $2 \mathrm{D}$ but not in $3 \mathrm{D}$, as a consequence of the asymptotics (2.9)). Thus it is difficult to envisage that the radiation condition (2.5) or (2.6) can hold in general in the case of $3 \mathrm{D}$ plane wave incidence.

Remark 5.6. In the 3D case, the above approach does apply to prove unique existence of solution in weighted spaces in the cases of incoming spherical or cylindrical waves. That is, it applies to the cases, respectively, where

$$
v^{i n}(\mathrm{x}):=\Phi(\mathrm{x}, \mathrm{z})=\frac{1}{4 \pi} \frac{\exp (i k|\mathrm{x}-\mathrm{z}|)}{|\mathrm{x}-\mathrm{z}|}, \quad \mathrm{x} \in \mathbb{R}^{3} \backslash\{\mathrm{z}\},
$$

for some $\mathrm{z} \in D$, and

$$
v^{i n}(\mathrm{x})=H_{0}^{(1)}\left(k \sqrt{x_{1}^{2}+H^{2}}\right), \quad \mathrm{x} \in \mathbb{R}^{3} \backslash \Sigma_{L},
$$

for some $H \in \mathbb{R}$ such that $\Sigma_{L}:=\left\{\left(0, x_{2}, H\right): x_{2} \in \mathbb{R}\right\} \subset D$. This second case is the case of an incident cylindrical wave generated by a line source occupying the line $\Sigma_{L}$ which, without loss of generality, we choose to lie in the plane $x_{1}=0$. The problem in each case is to find the unknown scattered field $v^{s c}$ and the total field $v=v^{s c}+v^{i n}$ such that

$$
\left(\Delta+k^{2}\right) v^{s c}=0 \text { in } D, \quad v=0 \text { on } \Gamma,
$$

and $v^{s c}$ satisfies an appropriate radiation condition. 
One way to make use of Theorem 4.1 to study these scattering problems is to formulate each scattering problem as an instance of (BVP) in section 2. To do this we can adapt the construction already used around (2.8). Precisely, in the spherical wave case, we choose $\epsilon>0$ such that $\operatorname{dist}(\mathrm{z}, \partial D)>\epsilon$ and replace $v^{\text {in }}$ by $\tilde{v}^{\text {in }}$ which coincides with $v^{\text {in }}$ except within distance $\epsilon$ of the source z, given by

$$
\tilde{v}^{i n}(\mathrm{x}):=\left\{\begin{array}{cc}
v^{i n}(\mathrm{x}), & |\mathrm{x}-\mathrm{z}|>\epsilon, \\
A+B \frac{\sin k r}{r}, & |\mathrm{x}-\mathrm{z}| \leq \epsilon,
\end{array}\right.
$$

where the constants $A$ and $B$ are chosen to ensure that $\tilde{v}^{i n} \in C^{1}\left(\mathbb{R}^{3}\right)$ (which is possible provided that $\epsilon$ is chosen sufficiently small, e.g., if $k \epsilon<1)$. Then $\tilde{v}^{i n} \in H_{l o c}^{2}\left(\mathbb{R}^{3}\right)$ with $\left(\Delta+k^{2}\right) v^{i n}=g_{S}$, where $g_{S}(\mathrm{x}):=A k^{2},|\mathrm{x}-\mathrm{z}|<\epsilon, g_{S}(\mathrm{x}):=0$ otherwise. Similarly, in the cylindrical wave case, we choose $\epsilon>0$ such that $\operatorname{dist}\left(\Sigma_{L}, \partial D\right)>\epsilon$ and replace $v^{\text {in }}$ by $\tilde{v}^{i n}$ which coincides with $v^{i n}$ except within distance $\epsilon$ of the source $\Sigma_{L}$, given by

$$
\tilde{v}^{i n}(\mathrm{x}):=\left\{\begin{array}{cl}
v^{i n}(\mathrm{x}), & \sqrt{x_{1}^{2}+\left(x_{3}-H\right)^{2}}>\epsilon, \\
A+B J_{0}\left(k \sqrt{x_{1}^{2}+\left(x_{3}-H\right)^{2}}\right), & \sqrt{x_{1}^{2}+\left(x_{3}-H\right)^{2}} \leq \epsilon,
\end{array}\right.
$$

where the constants $A$ and $B$ are chosen to ensure that $\tilde{v}^{i n} \in C^{1}\left(\mathbb{R}^{3}\right)$. (Again this is possible provided $\epsilon$ is chosen sufficiently small.) Then $\tilde{v}^{i n} \in H_{l o c}^{2}\left(\mathbb{R}^{3}\right)$ with $(\Delta+$ $\left.k^{2}\right) v^{i n}=g_{C}$, where $g_{C}(\mathrm{x}):=A k^{2}, \sqrt{x_{1}^{2}+\left(x_{3}-H\right)^{2}}<\epsilon, g_{C}(\mathrm{x}):=0$ otherwise. We observe that $g_{S}$ is compactly supported so that $g_{S} \in L_{\varrho}^{2}(D)$ for every $\varrho \in \mathbb{R}$. Further, it is an easy calculation to see that $g_{C} \in L_{\varrho}^{2}(D)$ for $\varrho<-1 / 2$ but not for $\varrho=-1 / 2$.

Since $v^{i n}=\tilde{v}^{i n}$, except in neighborhoods of $z$ or $\Sigma_{L}$ which do not intersect $\partial D$, so that, in particular, $v^{i n}=\tilde{v}^{i n}$ in a neighborhood of $\partial D$, the substitution of $v^{i n}$ by $\tilde{v}^{i n}$ does not change the scattered field $v^{s c}$. Further, since $\tilde{v}^{i n} \in H_{l o c}^{2}\left(\mathbb{R}^{3}\right)$, the scattering problem with this modified incident field can be formulated as an instance of (BVP). Precisely, in the spherical wave case, we can formulate the scattering problem as seeking the total field $\tilde{v}=\tilde{v}^{i n}+v^{s c}$ which satisfies (BVP) for some $\varrho \in(-1,1)$, with $g:=g_{S} \in L_{\varrho}^{2}(D)$ (and if $\operatorname{supp} g \not \subset S_{0}$, we need to replace $x_{n}$ by $x_{n}-a$ in (2.5) for some $a>0$ such that $\operatorname{supp} g \subset S_{a}$ ). Theorem 4.1 tells us that there is exactly one solution to this boundary value problem and that this solution $\tilde{v}$ satisfies $\left.\tilde{v}\right|_{S_{h}} \in V_{h, \varrho}$ for every $h \geq 0$ and $\varrho \in(-1,1)$. In the cylindrical wave case, we seek the total field $\tilde{v}=\tilde{v}^{i n}+v^{s c}$ which satisfies (BVP) for some $\varrho \in(-1,-1 / 2)$, with $g:=g_{C} \in L_{\varrho}^{2}(D)$ (again, if supp $g \not \subset S_{0}$, we need to replace $x_{n}$ by $x_{n}-a$ in (2.5) for some $a>0$ such that $\operatorname{supp} g \subset S_{a}$ ). Theorem 4.1 again tells us that there is exactly one solution and that this solution $\tilde{v}$ satisfies $\left.\tilde{v}\right|_{S_{h}} \in V_{h, \varrho}$ for every $h \geq 0$ and $\varrho \in(-1,-1 / 2)$.

5.2. Analysis of a finite section method for the variational formulation. An obvious approach to computing the solution to (BVP) numerically is to solve (V) by a finite element method. This is a standard approach for the numerical treatment of (DGPW) [20, 22], but in that case, the corresponding variational formulation, thanks to the periodicity, reduces to one on $S_{0}^{0}$, a single period of $S_{0}$. In the case of $(\mathrm{V})$, the region of integration is the whole infinite region $S_{0}$. Thus a necessary first step toward solving $(\mathrm{V})$ numerically is to approximate $(\mathrm{V})$ by a variational formulation on a domain of finite size to which standard FEMs can then be applied.

We are not aware of any analysis of such an approximation for $(\mathrm{V})$ or any similar variational formulations for rough surface scattering problems. However, the analogous approximation when boundary integral equation methods are applied to (BVP), namely, truncation of the region of integration, which is the infinite boundary $\partial D$, 
to a finite part of that boundary (a so-called finite section approximation) has been analyzed in both the 2D case [29] (and see [30, 23]) and, very recently, in the much more difficult 3D case [24]. In [29, 24], convergence and stability of modifications of the finite section method are proved. In the theses [30, 25] (cf. [16]), for the easier 2D case, convergence rates are also established via results on stability and convergence of the finite section method in weighted spaces of continuous functions.

In this section we prove stability and convergence of an approximation method in the same spirit for $(\mathrm{V})$. This approximation method consists simply of replacing $S_{0}$ by a finite region $S^{(R)} \subset S_{0}$ which coincides with $S_{0}$ in the region $|x|<R+1$ and of making the same approximation for $D$ so that $D$ is replaced by $D^{(R)} \subset D$ with $S^{(R)}=$ $D^{(R)} \backslash \bar{U}_{0}$. The only constraint on the choice of $S^{(R)}$ is that (2.1) and (2.2) should apply to $D^{(R)}$; this is the case, for example, for the simple explicit choice $S^{(R)}:=\{\mathrm{x}=$ $\left.\left(x, x_{n}\right) \in S_{0}:|x|<R+1\right\}$. In addition to proving stability and convergence, we also establish rates of convergence for the error measured in weighted spaces. In the case when $g$, the source of the acoustic waves, is compactly supported, these results imply that, locally in the energy norm, the error converges at a rate $O\left(R^{\epsilon-2}\right)$ for every $\epsilon>0$. This convergence rate is consistent with those obtained previously by methods specific to the 2D case for boundary integral equation formulations [30, 16, 23]. For example, the results in [30], in the case when the boundary $\Gamma$ is the graph of a function which is sufficiently smooth, imply a convergence rate $R^{-2}$ locally in the uniform norm for the solution of a boundary integral formulation when the source of the acoustic waves is compactly supported and the finite section that is taken coincides with $\Gamma$ inside a ball of radius $R$.

Given $g \in L_{\varrho}^{2}\left(S_{0}\right)$, with $|\varrho|<1$, let $u \in V_{\varrho}$ be the unique solution of the variational problem (V) so that

$$
B(u, v)=-(g, v) \text { for all } v \in V_{-\varrho} .
$$

For $R>0$, we approximate problem (5.11) by a corresponding variational equation on the bounded domain $S^{(R)}$. For $\varrho \in \mathbb{R}$ and $R>0$, let $V_{\varrho}^{(R)}$ denote the Hilbert space $V_{\varrho}$ in the case that we replace $D$ by $D^{(R)}$; explicitly $V_{\varrho}^{(R)}$ denotes the completion of $\left\{\left.u\right|_{S_{0}^{(R)}}: u \in C_{0}^{\infty}\left(D^{(R)}\right)\right\}$ in the norm

$$
\|u\|_{V_{\varrho}^{(R)}}=\left(\int_{S_{0}^{(R)}}\left(\left|\left(1+x^{2}\right)^{\varrho / 2} u\right|^{2}+\left|\nabla\left(\left(1+x^{2}\right)^{\varrho / 2} u\right)\right|^{2}\right) d \mathrm{x}\right)^{1 / 2} .
$$

We remark, as is easily seen from Lemma 2.1 , that the norms $\|\cdot\|_{V_{\varrho}^{(R)}}, \varrho \in \mathbb{R}$, are equivalent since $S_{0}^{(R)}$ is bounded so that, as linear spaces, for $\varrho \in \mathbb{R}, V_{\varrho}^{(R)}=V^{(R)}:=$ $V_{0}^{(R)}$. The approximating variational problem is the following: find $u^{(R)} \in V^{(R)}$ such that

$$
B^{(R)}\left(u^{(R)}, v\right)=-(g, v) \quad \text { for all } v \in V^{(R)} .
$$

Here $B^{(R)}$ is the continuous sesquilinear form on $V^{(R)} \times V^{(R)}$ defined by (3.3) with $D$ replaced by $D^{(R)}$, i.e., defined by

$$
B^{(R)}(u, v):=\int_{S_{0}^{(R)}}\left(\nabla u \cdot \nabla \bar{v}-k^{2} u \bar{v}\right) d \mathrm{x}+\int_{\Gamma_{0}^{(R)}} \gamma_{-} \bar{v} T \gamma_{-} u d s(x),
$$

where $\Gamma_{0}^{(R)}:=\overline{S_{0}^{(R)}} \cap \Gamma_{0}$ (see Remark 3.6 for the interpretation of $\gamma_{-}$in this case). 
Making the observation that we can view $V_{\varrho}^{(R)}$ as a closed subspace of $V_{\varrho}$ (the elements of $V_{\varrho}^{(R)}$ become elements of $V_{\varrho}$ if we extend them by zero from $S_{0}^{(R)}$ to $S_{0}$ ), the analysis of the error in approximating $u$ by $u^{(R)}$ follows the usual pattern for analyzing the Galerkin method for variational problems via a generalized Céa's lemma. Precisely, if $\tilde{u} \in V^{(R)} \subset V_{\varrho}$, then, for $v \in V^{(R)}$, applying (5.11),

$$
B^{(R)}(\tilde{u}, v)=B(\tilde{u}, v)=B(\tilde{u}-u, v)-(g, v) .
$$

Subtracting this equation from (5.13), we see that

$$
B^{(R)}\left(\tilde{u}-u^{(R)}, v\right)=B(\tilde{u}-u, v) \quad \text { for all } v \in V^{(R)} .
$$

Now recall from section 4 that $\mathcal{B}_{\varrho}: V_{\varrho} \rightarrow V_{-\varrho}^{*}$ is our notation for the bounded linear operator induced by the continuous sesquilinear form $B$. Similarly, let $\mathcal{B}_{\varrho}^{(R)}: V_{\varrho}^{(R)} \rightarrow$ $V_{-\varrho}^{(R)^{*}}$ denote the operator induced by the sesquilinear form $B^{(R)}$; in other words, $\mathcal{B}_{\varrho}^{(R)}$ is just $\mathcal{B}_{\varrho}$ in the case that $D$ is replaced by $D^{(R)}$. From Theorem 4.1 it is clear that $\mathcal{B}_{\varrho}^{(R)}$ is invertible for every $R>0$ and $\varrho \in(-1,1)$. From Remark 4.2 it is clear, moreover, that $\left\|\left(\mathcal{B}_{\varrho}^{(R)}\right)^{-1}\right\|$ is bounded uniformly for $R>0$, with a bound which depends only on $|b|, k$, and $\varrho$. Thus, from (5.15), it follows that, for $\varrho_{1} \in(-1,1)$,

$$
\left\|\tilde{u}-u^{(R)}\right\|_{V_{\varrho_{1}}^{(R)}} \leq c\|\tilde{u}-u\|_{V_{\varrho_{1}}}
$$

where the constant $c>0$, which depends only on $|b|, k$, and $\varrho_{1}$, is an upper bound for $\left\|\mathcal{B}_{\varrho_{1}}\right\| \sup _{R>0}\left\|\left(\mathcal{B}_{\varrho_{1}}^{(R)}\right)^{-1}\right\|$. Thus

$$
\left\|u-u^{(R)}\right\|_{V_{\varrho_{1}}} \leq(1+c) \inf _{\tilde{u} \in V_{\varrho_{1}}^{(R)}}\|\tilde{u}-u\|_{V_{\varrho_{1}}} .
$$

To obtain a more concrete error estimate, choose a cutoff function $\chi_{R} \in$ $C_{0}^{\infty}\left(\mathbb{R}^{n-1}\right)$ such that, for all $R>0$,

$$
\chi_{R}(x):=1 \text { for }|x|<R, \quad \chi_{R}(x):=0 \text { for }|x|>R+1, \quad \sup _{\mathbb{R}^{n-1}}\left\{\left|\chi_{R}\right|+\left|\nabla \chi_{R}\right|\right\} \leq c_{1}
$$

for some constant $c_{1}>0$ independent of $R$. Defining $\tilde{u} \in V_{\varrho_{1}}^{(R)}$ by $\tilde{u}(\mathrm{x}):=u(\mathrm{x}) \chi_{R}(x)$, $\mathrm{x} \in S_{0}$, we see by using Lemma 2.1 that, for $-1<\varrho_{1} \leq \varrho$, where $\widetilde{S}_{0}^{R}:=\left\{\mathrm{x} \in S_{0}\right.$ : $|x|>R\}$ and $c_{2}, c_{3}$, and $c_{4}$ denote further constants dependent only on $\varrho$ and $|b|$,

$$
\begin{aligned}
\|\tilde{u}-u\|_{V_{\varrho_{1}}} & =\left\|\left(1-\chi_{R}\right) u\right\|_{V_{\varrho_{1}}} \\
& \leq c_{2}\left(\int_{\tilde{S}_{0}^{R}}\left(1+x^{2}\right)^{\varrho_{1}}\left(|u|^{2}+|\nabla u|^{2}\right) d \mathrm{x}\right)^{1 / 2} \\
& \leq c_{3} R^{\varrho_{1}-\varrho}\left(\int_{\tilde{S}_{0}^{R}}\left(1+x^{2}\right)^{\varrho}\left(|u|^{2}+|\nabla u|^{2}\right) d \mathrm{x}\right)^{1 / 2} \\
& \leq c_{4} R^{\varrho_{1}-\varrho}\|u\|_{V_{\varrho}} .
\end{aligned}
$$

We see that we have proved the following theorem.

TheOrem 5.7. Suppose $g \in L_{\varrho}^{2}\left(S_{0}\right)$ with $|\varrho|<1$, and let $u \in V_{\varrho}$ be the unique solution of the variational problem $(V)$. Choose, for $R>0$, approximating domains 
$D^{(R)} \subset D$ which satisfy (2.1) and (2.2) and are such that $S^{(R)} \subset S_{0}$ is bounded and $S^{(R)} \supset \Omega_{R}:=\left\{\mathrm{x} \in S_{0}:|x|<R+1\right\}$. Then the approximating variational problem (5.11) on the finite region $S^{(R)}$ has exactly one solution $u^{(R)}$ for every $R>0$. Further, for some constant $c>0$ dependent only on $k, \varrho, \varrho_{1}$, and $|b|$, it holds for $-1<\varrho_{1}<\varrho$ that

$$
\left\|u-u^{(R)}\right\|_{V_{\varrho_{1}}^{(R)}} \leq c R^{\varrho_{1}-\varrho}\|u\|_{V_{\varrho}} .
$$

As a consequence, for every $R_{1}>0$, it holds that $\left\|u-u^{(R)}\right\|_{H^{1}\left(\Omega_{R_{1}}\right)}=O\left(R^{\varrho_{1}-\varrho}\right)$ as $R \rightarrow \infty$. In particular, if $g \in L_{\varrho}^{2}\left(S_{0}\right)$ for every $\varrho<1$, which holds, for example, if $g$ is compactly supported, then, for every $\epsilon>0$ and $R_{1}>0$,

$$
\left\|u-u^{(R)}\right\|_{H^{1}\left(\Omega_{R_{1}}\right)}=O\left(R^{\epsilon-2}\right) \quad \text { as } \quad R \rightarrow \infty .
$$

6. Commutator estimates. This section is devoted to the proofs of Theorem 3.1 and Lemma 3.4 (i). Let $k>0, a>0$, and consider the pseudodifferential operator $T_{a}$ on $\mathbb{R}^{m}, m:=n-1=1,2$, with symbol $t_{a}(\xi)$ :

$$
T_{a} u(x)=F^{-1} t_{a}(\xi) F u(\xi), \quad t_{a}(\xi):=a^{-1} \sqrt{\xi^{2}-k^{2} a^{2}} .
$$

Here and in the following the square root is chosen so that its argument lies in $[-\pi / 2,0]$ :

(6.2) $t_{a}(\xi)=(-i / a) \sqrt{k^{2} a^{2}-\xi^{2}},|\xi| \leq k a ; \quad t_{a}(\xi)=(1 / a) \sqrt{\xi^{2}-k^{2} a^{2}},|\xi|>k a$.

We have $T_{1}=T, t_{1}=t$, where $T$ and $t$ are defined in (3.1).

Using a scaling argument, we reduce the assertion of Theorem 3.1 to a corresponding estimate for the commutator defined by

$$
C_{a}:=T_{a}-\left(1+x^{2}\right)^{\varrho / 2} T_{a}\left(1+x^{2}\right)^{-\varrho / 2} .
$$

With $S_{a} u(x):=u(a x)$, we obtain $F S_{a} u=a^{-m} S_{1 / a} F u$, and the same relation holds with $F$ replaced by $F^{-1}$. Hence

$$
a^{m} S_{a} F^{-1} t F u=F^{-1}\left(S_{1 / a} t\right) S_{1 / a} F u=a^{m} F^{-1}\left(S_{1 / a} t\right) F S_{a} u,
$$

giving $S_{a} T u=T_{a} S_{a} u$, where $T_{a}$ is the operator (6.1) with the symbol $t(\xi / a)=t_{a}(\xi)$. From (3.2) and (6.3), we then have

$$
S_{a} C=T_{a} S_{a}-\left(1+x^{2}\right)^{\varrho / 2} T_{a}\left(1+x^{2}\right)^{-\varrho / 2} S_{a}=C_{a} S_{a} .
$$

Using the relation $\left\|S_{a} u\right\|_{L^{2}\left(\mathbb{R}^{m}\right)}=a^{-m / 2}\|u\|_{L^{2}\left(\mathbb{R}^{m}\right)}$ and (6.4), we now observe that Theorem 3.1 is equivalent to the following theorem.

TheOREm 6.1. For $k a \geq 1$ and $|\varrho|<1$, the commutator $C_{a}$ defined in (6.3) has norm $\leq c(\varrho) \sqrt{k / a}$ on $L^{2}\left(\mathbb{R}^{m}\right)$.

It is enough to consider $\varrho \in(0,1)$ since the case of negative $\varrho$ then follows by duality (with respect to the scalar product on $L^{2}\left(\mathbb{R}^{m}\right)$ ). We split the symbol $t_{a}$ as

$$
t_{a}=t^{(0)}+t^{(1)}=: \chi(|\xi|) t_{a}(\xi)+(1-\chi(|\xi|)) t_{a}(\xi)
$$

where $\chi$ is a suitable cutoff funtion (see below), and we consider the corresponding decomposition of $T_{a}$,

$$
T_{a}=T^{(0)}+T^{(1)}
$$

Copyright (c) by SIAM. Unauthorized reproduction of this article is prohibited. 
where $T^{(j)}$ is defined by (6.1) with $t^{(j)}$ in place of $t_{a}$. Then the commutator $C_{a}$ takes the form

$$
\begin{aligned}
C_{a}=C^{(0)}+C^{(1)} & =:\left(T^{(0)}-\left(1+x^{2}\right)^{\varrho / 2} T^{(0)}\left(1+x^{2}\right)^{-\varrho / 2} \cdot\right) \\
& +\left(T^{(1)}-\left(1+x^{2}\right)^{\varrho / 2} T^{(1)}\left(1+x^{2}\right)^{-\varrho / 2} \cdot\right) .
\end{aligned}
$$

We will estimate the norm of the operators

$$
\begin{aligned}
N:=\left(1+x^{2}\right)^{-\varrho / 2} C^{(0)}: L^{2}\left(\mathbb{R}^{m}\right) & \rightarrow L_{\varrho}^{2}\left(\mathbb{R}^{m}\right), \\
\hat{N} u:=F N F^{-1} u, \quad \hat{N}: L^{2}\left(\mathbb{R}^{m}\right) & \rightarrow H^{\varrho}\left(\mathbb{R}^{m}\right), \\
\hat{T}:=t^{(1)}(\xi) \cdot: H^{\varrho}\left(\mathbb{R}^{m}\right) & \rightarrow H^{\varrho}\left(\mathbb{R}^{m}\right) .
\end{aligned}
$$

In view of (6.7)-(6.10) and recalling that $F$ is an isometry of $L_{\varrho}^{2}\left(\mathbb{R}^{m}\right)$ onto $H^{\varrho}\left(\mathbb{R}^{m}\right)$ for every $\varrho \in \mathbb{R}$, Theorem 6.1 then follows from Theorem 6.2.

TheOREM 6.2. (i) For $\varrho \in(0,1]$, the norm of $\hat{N}$ is bounded by $c(\varrho) \sqrt{k / a}$.

(ii) For $\varrho \in[0,1)$, the norm of $\hat{T}$ is bounded by $c(\varrho) \sqrt{k / a}$, too.

We now choose the cutoff function $\chi \in C^{\infty}[0, \infty)$ with $0 \leq \chi \leq 1$ and

$$
\chi(r)=0 \quad \text { on } \quad|r-k a| \leq 1 / 3, \quad \chi(r)=1 \quad \text { on } \quad|r-k a| \geq 2 / 3,
$$

and such that, for some $c>0$ independent of $k a \geq 1$,

$$
\left|\partial_{r} \chi(r)\right| \leq c \quad \text { on } \quad \mathbb{R}^{+} .
$$

Note that (6.11) implies

$$
\partial_{r} \chi(r)=0 \quad \text { on } \quad\{|r-k a| \leq 1 / 3\} \cup\{|r-k a| \geq 2 / 3\} .
$$

To prove Theorem 6.2 , we need some auxiliary results.

Lemma 6.3. For $k a \geq 1$, we have $\left|\partial_{r} t^{(0)}(\xi)\right| \leq c \sqrt{k / a}$ on $\mathbb{R}^{+}$, where $r=|\xi|$.

Proof. Setting $h(r):=a t_{a}(\xi)=(r-k a)^{1 / 2}(r+k a)^{1 / 2}$ for $r>k a$ and $h(r):=$ iat $_{a}(\xi)=(k a-r)^{1 / 2}(r+k a)^{1 / 2}$ for $r<k a$, we obtain

$$
\partial_{r} h=\left\{\begin{array}{cc}
r / h, & r>k a, \\
-r / h, & 0 \leq r<k a,
\end{array} \quad \partial_{r}^{2} h=-k^{2} a^{2} / h^{3}, \quad r \neq k a,\right.
$$

which implies that $\partial_{r}^{j} h(j=1,2)$ do not change sign on $(0, k a)$ and $(k a, \infty)$. Therefore, the maximum of $h$ on $1 / 3 \leq|r-k a| \leq 2 / 3$ is attained at $r=k a-2 / 3$ or $r=k a+2 / 3$, while the maximum of $\left|\partial_{r} h\right|$ on $|r-k a| \geq 1 / 3$ is attained at $r=k a-1 / 3$ or $r=$ $k a+1 / 3$, and both maxima are bounded from above by $c \sqrt{k a}$. Together with (6.11)(6.13), this easily implies the result.

To prove Theorem 6.2 (i), we write (cf. (6.7)-(6.9))

$$
\hat{N} F u(\xi)=\int_{\mathbb{R}^{m}} b_{\varrho}(\xi-\eta)\left(t^{(0)}(\eta)-t^{(0)}(\xi)\right) F u(\eta) d \eta, \quad u \in C_{0}^{\infty}\left(\mathbb{R}^{m}\right),
$$

with $b_{\varrho}:=F\left(1+x^{2}\right)^{-\varrho / 2}$. Here the integral in (6.14) is well defined since $F u$ is rapidly decreasing and $b_{\varrho} \in L^{1}\left(\mathbb{R}^{m}\right)$ for $\varrho>0$ (see the next lemma), and we have used the relation $F\left(1+x^{2}\right)^{-\varrho / 2} v=b_{\varrho} * F v$ for a function $v$ of rapid decay, with $*$ denoting convolution.

LEMma 6.4. For any $\varrho>0$, the functions $b_{\varrho}$ and $|\xi| \nabla_{\xi} b_{\varrho}$ are rapidly decreasing as $|\xi| \rightarrow \infty$ and belong to $L^{1}\left(\mathbb{R}^{m}\right)$. 
For the proof of this, we refer to [32, Chap. 8.1]; see also [37, Chap. 5.3].

Proof of Theorem 6.2 (i). From (6.14) and Lemma 6.3,

$$
\begin{aligned}
\|\hat{N} F u\|_{L^{2}\left(\mathbb{R}^{m}\right)} & \leq\left\|\int_{\mathbb{R}^{m}}\left|b_{\varrho}(\xi-\eta)\right||\xi-\eta| \sup _{\mathbb{R}^{+}}\left|\partial_{r} t^{(0)}\right||F u(\eta)| d \eta\right\|_{L^{2}\left(\mathbb{R}^{m}\right)} \\
& \leq c \sqrt{k / a}\left\||\xi| b_{\varrho}\right\|_{L^{1}\left(\mathbb{R}^{m}\right)}\|F u\|_{L^{2}\left(\mathbb{R}^{m}\right)}
\end{aligned}
$$

using the mean value theorem and Young's inequality. Moreover, since

$$
\begin{aligned}
\nabla_{\xi} \hat{N} F u(\xi) & =\int_{\mathbb{R}^{m}}\left(t^{(0)}(\eta)-t^{(0)}(\xi)\right) \nabla_{\xi} b_{\varrho}(\xi-\eta) F u(\eta) d \eta \\
& +\int_{\mathbb{R}^{m}} b_{\varrho}(\xi-\eta)\left(-\nabla_{\xi} t^{(0)}(\xi)\right) F u(\eta) d \eta
\end{aligned}
$$

we obtain analogously

$$
\|\nabla \hat{N} F u\|_{L^{2}\left(\mathbb{R}^{m}\right)} \leq c \sqrt{k / a}\left(\left\||\xi| \nabla b_{\varrho}\right\|_{L^{1}\left(\mathbb{R}^{m}\right)}+\left\|b_{\varrho}\right\|_{L^{1}\left(\mathbb{R}^{m}\right)}\right)\|F u\|_{L^{2}\left(\mathbb{R}^{m}\right)} .
$$

Together with Lemma $6.4,(6.15)$ and (6.16) imply that, for any $\varrho \in(0,1]$, the operators $\hat{N}: L^{2}\left(\mathbb{R}^{m}\right) \rightarrow L^{2}\left(\mathbb{R}^{m}\right)$ and $\hat{N}: L^{2}\left(\mathbb{R}^{m}\right) \rightarrow H^{1}\left(\mathbb{R}^{m}\right)$ have norm $\leq c(\varrho) \sqrt{k / a}$. By interpolation, we then get the result.

Proof of Theorem 6.2 (ii). We have to show that the multiplication operator

$$
\hat{T} v=(1-\chi(|\xi|)) a^{-1} \sqrt{\xi^{2}-k^{2} a^{2}} v: H^{\varrho}\left(\mathbb{R}^{m}\right) \rightarrow H^{\varrho}\left(\mathbb{R}^{m}\right), \quad 0 \leq \varrho<1,
$$

has norm $\leq c(\varrho) \sqrt{k / a}$. Note that the support of $1-\chi$ is contained in the set $\mathcal{R}:=$ $\{|r-k a| \leq 2 / 3\}, r=|\xi|$; see (6.11). By localization, (6.17) can be reduced to an estimate of the form

$$
\|q v\|_{H^{\varrho}\left(\mathbb{R}^{m}\right)} \leq c(\varrho) \sqrt{k / a}\|v\|_{H^{\varrho}\left(\mathbb{R}^{m}\right)}, \quad 0 \leq \varrho<1,
$$

where

$$
q(\xi):=a^{-1} \sqrt{\xi_{m}}\left(\xi_{m}+2 k a\right)^{1 / 2} \psi\left(\xi_{m}\right)
$$

with $\psi \in C_{0}^{\infty}(-2 / 3,2 / 3)$ fixed and $\sqrt{\xi_{m}}=-i\left|\xi_{m}\right|^{1 / 2}$ for $\xi_{m}<0$. This reduction is clear for $m=1$, where we have to localize near $\xi_{1}=k a$ and $\xi_{1}=-k a$. For $m=2$, we parametrize the annulus $\mathcal{R}$ by $\xi_{2}:=r-k a$ and arclength $\xi_{1}$ on $|\xi|=r=k a$ and need two local charts again to cover $\mathcal{R}$. Note that the Jacobians of the corresponding coordinate transformations (with respect to the original $\xi$-coordinates) are uniformly bounded from above and below for $k a \geq 1$. We omit the details since we present an alternative approach in the $3 \mathrm{D}$ case below.

To prove (6.18), we first observe that the operator of multiplication by $q_{1}:=$ $a^{-1}\left(\xi_{m}+2 k a\right)^{1 / 2} \psi$ has norm

$$
\sup _{\mathbb{R}}\left|q_{1}\right| \leq c \sqrt{k / a} \quad \text { and } \quad \leq \sup _{\mathbb{R}}\left|q_{1}\right|+\sup _{\mathbb{R}}\left|\partial_{m} q_{1}\right| \leq c \sqrt{k / a}
$$

in $L^{2}\left(\mathbb{R}^{m}\right)$ and $H^{1}\left(\mathbb{R}^{m}\right)$, respectively. Note that $k a \geq 1$ implies $1 / a \leq k$ and

$$
a^{-1}\left(\xi_{m}+2 k a\right)^{-1 / 2}|\psi| \leq c a^{-1} / \sqrt{k a} \leq c \sqrt{k / a} .
$$

Copyright $@$ by SIAM. Unauthorized reproduction of this article is prohibited. 
By interpolation, the norm of this multiplication operator in $H^{\varrho}\left(\mathbb{R}^{m}\right)$ is then bounded by $c(\varrho) \sqrt{k / a}$. It remains to show that

$$
\left\|\sqrt{\xi_{m}} \varphi\left(\xi_{m}\right) v\right\|_{H^{\varrho}\left(\mathbb{R}^{m}\right)} \leq c(\varrho)\|v\|_{H^{\varrho}\left(\mathbb{R}^{m}\right)}, \quad 0 \leq \varrho<1,
$$

where $\varphi$ is a smooth function with somewhat larger support and $\varphi \psi=\psi$.

Let first $m=1$. Then (6.20) follows for $\varrho \in(1 / 2,1)$ since $\sqrt{\xi_{1}} \varphi \in H^{\varrho}(\mathbb{R})$ for $\varrho<1$ (but not for $\varrho=1$ ) and $H^{\varrho}(\mathbb{R})$ is a Banach algebra. Since (6.20) is obvious for $\varrho=0$, we obtain the result by interpolation. Note that the constant $c(\varrho)$ blows up as $\varrho \rightarrow 1$.

For $m=2$, the proof of (6.20) can easily be reduced to the case $m=1$ by using the relation (cf. [28, Chap. 1])

$$
H^{\varrho}\left(\mathbb{R}^{2}\right)=L^{2}\left(\mathbb{R} ; H^{\varrho}(\mathbb{R})\right) \cap H^{\varrho}\left(\mathbb{R} ; L^{2}(\mathbb{R})\right)
$$

and the fact that the function (6.19) is independent of $\xi_{1}$.

Remark 6.5. An alternative proof of Theorem 6.2 (ii) for $m=2$ can be given by the following more direct reduction to the case $m=1$. Let $(r, \theta)$ be polar coordinates in $\mathbb{R}^{2}$, and consider a multiplication operator $\mathcal{M}:=q \cdot$ on $H^{\varrho}\left(\mathbb{R}^{2}\right), \varrho \in(0,1]$, with a continuous function $q=q(r)$ depending only on the radial variable, $\operatorname{supp} q \subset[1 / 3,2 / 3]$, and such that $q$. considered as a multiplication operator on $H^{\varrho}(\mathbb{R})$ is bounded with norm $\|q \cdot\|_{\varrho}$. Then the norm of $\mathcal{M}$ on $H^{\varrho}\left(\mathbb{R}^{2}\right)$ is bounded by a positive constant $c(\varrho)$. Applying this to the operator $\hat{T}$ defined in (6.17) and using Theorem 6.2 (ii) for $m=1$, we get the result for $m=2$.

To prove the above norm estimate for $\mathcal{M}$, we first note that $L^{2}\left(\mathbb{R}^{2}\right)$ is the orthogonal sum of the subspaces

$$
\mathcal{H}_{j}:=\left\{v \in L^{2}\left(\mathbb{R}^{2}\right): v=f(r) \exp (i j \theta), \quad \int_{0}^{\infty}|f(r)|^{2} r d r<\infty\right\}, \quad j \in \mathbb{Z},
$$

and the Fourier transform leaves each space $\mathcal{H}_{j}$ invariant; see [38, Chap. 4.1]. Therefore, it is sufficient to verify that

$$
\|q f \exp (i j \theta)\|_{H^{\varrho}\left(\mathbb{R}^{2}\right)} \leq c(\varrho)\|f \exp (i j \theta)\|_{H^{\varrho}\left(\mathbb{R}^{2}\right)}
$$

for each $j \in \mathbb{Z}$ and $f \in C_{0}^{\infty}(0, \infty)$. Furthermore, we have, uniformly in $j$,

$$
\begin{aligned}
\|q f \exp (i j \theta)\|_{L^{2}\left(\mathbb{R}^{2}\right)} & \sim\left\|r^{1 / 2} q f\right\|_{L^{2}(\mathbb{R})}, \\
\|q f \exp (i j \theta)\|_{H^{1}\left(\mathbb{R}^{2}\right)} & \sim\|q f \exp (i j \theta)\|_{L^{2}\left(\mathbb{R}^{2}\right)} \\
& +\left\|\partial_{r} q f \exp (i j \theta)\right\|_{L^{2}\left(\mathbb{R}^{2}\right)}+\left\|q f r^{-1} \partial_{\theta} \exp (i j \theta)\right\|_{L^{2}\left(\mathbb{R}^{2}\right)} \\
& \sim\left\|r^{1 / 2} q f\right\|_{H^{1}(\mathbb{R})}+(1+|j|)\left\|r^{-1 / 2} q f\right\|_{L^{2}(\mathbb{R})},
\end{aligned}
$$

and thus, by interpolation,

$$
\|q f \exp (i j \theta)\|_{H^{\varrho}\left(\mathbb{R}^{2}\right)} \sim\left\|r^{1 / 2} q f\right\|_{H^{\varrho}(\mathbb{R})}+(1+|j|)^{\varrho}\left\|r^{1 / 2-\varrho} q f\right\|_{L^{2}(\mathbb{R})},
$$

where $\sim$ means equivalence of norms. Here we used a standard interpolation of Sobolev norms and the interpolation theorem for weighted $L^{2}$ spaces (see [40, Chap. 1.18.5]). Now $(6.22)$ and the boundedness of $q \cdot$ on $H^{\varrho}(\mathbb{R})$ imply the estimates

$$
\begin{aligned}
\|q f \exp (i j \theta)\|_{H^{\varrho}\left(\mathbb{R}^{2}\right)} & \leq c(\varrho)\left\{\left\|q r^{1 / 2} f\right\|_{H \varrho(\mathbb{R})}+(1+|j|)^{\varrho}\left\|q r^{1 / 2-\varrho} f\right\|_{L^{2}(\mathbb{R})}\right\} \\
& \leq c(\varrho) \max \left(\|q \cdot\|_{\varrho}, \sup |q|\right)\left\{\left\|r^{1 / 2} f\right\|_{H^{\varrho}(\mathbb{R})}+(1+|j|)^{\varrho}\left\|r^{1 / 2-\varrho} f\right\|_{L^{2}(\mathbb{R})}\right\} \\
& \leq c(\varrho) \max \left(\|q \cdot\|_{\varrho}, \sup |q|\right)\|f \exp (i j \theta)\|_{H^{\varrho}\left(\mathbb{R}^{2}\right)},
\end{aligned}
$$

Copyright $\odot$ by SIAM. Unauthorized reproduction of this article is prohibited. 
giving (6.21).

Remark 6.6. (i) Repeating the above proofs with a fixed cutoff function $\chi$ vanishing in a neighborhood of $|\xi|=k$, we obtain the norm estimate of Lemma 3.2 for the commutator $T-\left(1+x^{2}\right)^{\varrho / 2} T\left(1+x^{2}\right)^{-\varrho / 2}$. Here we need not take care of the dependence of the constants on $a$ and $k$.

(ii) Note that the symbol $t^{(0)}=\chi t$ of the pseudodifferential operator $T^{(0)}=$ $F^{-1} t^{(0)} F$ is a smooth function satisfying $\left|t_{0}\right| \leq c\left(1+\xi^{2}\right)^{1 / 2}$ and $\left|\nabla t_{0}\right| \leq c$ on $\mathbb{R}^{m}$, and this is enough to obtain the boundedness of the commutators $T^{(0)}-\left(1+x^{2}\right)^{\varrho / 2} T^{(0)}(1+$ $\left.x^{2}\right)^{-\varrho / 2} \cdot, \varrho \mid \leq 1$, on $L^{2}\left(\mathbb{R}^{m}\right)$; see the estimates (6.15) and (6.16).

Applying this to the operator $\Lambda:=F^{-1}\left(1+\xi^{2}\right)^{1 / 2} F$ which is an isomorphism of $H^{1}\left(\mathbb{R}^{m}\right)$ onto $L^{2}\left(\mathbb{R}^{m}\right)$, we observe that $\Lambda$ is also an isomorphism of $H_{\varrho}^{1}\left(\mathbb{R}^{m}\right)$ onto $L_{\varrho}^{2}\left(\mathbb{R}^{m}\right)$ and that $F$ is an isomorphism of $H_{\varrho}^{1}\left(\mathbb{R}^{m}\right)$ onto $H_{1}^{\varrho}\left(\mathbb{R}^{m}\right)$, at least for $|\varrho| \leq 1$. This is also true for arbitrary $\varrho \in \mathbb{R}$; see [34] and [41].

(iii) Let $\sigma(\xi)$ be a smooth symbol satisfying the estimates $|\sigma| \leq c,\left|\nabla_{\xi} \sigma\right| \leq$ $c\left(1+\xi^{2}\right)^{-1 / 2}$ on $\mathbb{R}^{m}$. Then, for $A:=F^{-1} \sigma F$ and $|\varrho| \leq 1$, the commutator

$$
A-\left(1+x^{2}\right)^{\varrho / 2} A\left(1+x^{2}\right)^{-\varrho / 2} \cdot: L^{2}\left(\mathbb{R}^{m}\right) \rightarrow H^{1}\left(\mathbb{R}^{m}\right)
$$

is bounded. This follows from (ii) applied to the operator $B=\Lambda A$ with the symbol $\left(1+\xi^{2}\right)^{1 / 2} \sigma(\xi)$ and the relation

$$
\begin{aligned}
\Lambda^{-1} B & -\left(1-x^{2}\right)^{\varrho / 2} \Lambda^{-1} B\left(1+x^{2}\right)^{-\varrho / 2} \cdot=\Lambda^{-1}\left(B-\left(1+x^{2}\right)^{\varrho / 2} B\left(1+x^{2}\right)^{-\varrho / 2} \cdot\right) \\
& +\left(\Lambda^{-1}-\left(1+x^{2}\right)^{\varrho / 2} \Lambda^{-1}\left(1+x^{2}\right)^{-\varrho / 2} \cdot\right)\left(1+x^{2}\right)^{\varrho / 2} B\left(1+x^{2}\right)^{-\varrho / 2} \cdot .
\end{aligned}
$$

Note that $B$ is bounded on $L_{\varrho}^{2}\left(\mathbb{R}^{m}\right)$.

More general results on pseudodifferential operators with smooth symbols in weighted Sobolev spaces can be found in [34] and [41].

Finally, we proceed to the proof of Lemma 3.4.

Proof of Lemma 3.4 (i). From (2.5) we have, for $u_{0} \in C_{0}^{\infty}\left(\Gamma_{0}\right)$,

$$
\begin{aligned}
u\left(x, x_{n}\right) & =F^{-1} \exp \left(-x_{n} t(\xi)\right) F u_{0}=: M_{0} u_{0}, \quad t(\xi):=\sqrt{\xi^{2}-k^{2}}, \\
\nabla_{x} u\left(x, x_{n}\right) & =F^{-1} i \xi \exp \left(-x_{n} t(\xi)\right) F u_{0}=: M_{1} u_{0}=\nabla_{x} M_{0} u_{0}, \\
\partial_{n} u\left(x, x_{n}\right) & =F^{-1}(-t(\xi)) \exp \left(-x_{n} t(\xi)\right) F u_{0}=: M_{2} u_{0}=-T M_{0} u_{0} .
\end{aligned}
$$

We have to prove the estimates, for $|\varrho|<1$ and $h>0$,

$$
\|u\|_{H_{\varrho}^{1}\left(U_{0} \backslash \bar{U}_{h}\right)} \leq c(h, \varrho)\left\|u_{0}\right\|_{H_{\varrho}^{1 / 2}\left(\Gamma_{0}\right)}, \quad u_{0} \in C_{0}^{\infty}\left(\Gamma_{0}\right),
$$

or equivalently, with $m=n-1$,

$$
\int_{0}^{h} \int_{\mathbb{R}^{m}}\left(1+x^{2}\right)^{\varrho} \sum_{j=0}^{2}\left|M_{j} u_{0}\right|^{2} d x d x_{n} \leq c(h, \varrho)\left\|\left(1+x^{2}\right)^{\varrho / 2} u_{0}\right\|_{H^{1 / 2}\left(\mathbb{R}^{m}\right)}^{2} .
$$

This was proved in [11] for $\varrho=0$ by taking the Fourier transform. To verify (6.25) for $\varrho \neq 0$, it is then sufficient to show that the commutators

$$
M_{j}-\left(1+x^{2}\right)^{\varrho / 2} M_{j}\left(1+x^{2}\right)^{-\varrho / 2} ., \quad j=0,1,2,
$$

are uniformly bounded on $L^{2}\left(\mathbb{R}^{m}\right)$ with respect to $x_{n} \in(0, h)$; compare the proof of Lemma 3.3 (ii). We can write (cf. relation (6.23))

$$
\begin{aligned}
A M_{0} & -\left(1+x^{2}\right)^{\varrho / 2} A M_{0}\left(1+x^{2}\right)^{-\varrho / 2} \cdot=A\left(M_{0}-\left(1+x^{2}\right)^{\varrho / 2} M_{0}\left(1+x^{2}\right)^{-\varrho / 2} \cdot\right) \\
& +\left(A-\left(1+x^{2}\right)^{\varrho / 2} A\left(1+x^{2}\right)^{-\varrho / 2} \cdot\right)\left(1+x^{2}\right)^{\varrho / 2} M_{0}\left(1+x^{2}\right)^{-\varrho / 2} \cdot
\end{aligned}
$$

Copyright $@$ by SIAM. Unauthorized reproduction of this article is prohibited. 
where $A$ is one of the operators $\partial_{j}=\partial / \partial x_{j}, 1 \leq j \leq m$, and $T$. Therefore, it is enough to prove the uniform boundedness of

$$
\begin{gathered}
M_{0}: L_{\varrho}^{2}\left(\mathbb{R}^{m}\right) \rightarrow L_{\varrho}^{2}\left(\mathbb{R}^{m}\right), \quad|\varrho|<1, \\
M_{0}-\left(1+x^{2}\right)^{\varrho / 2} M_{0}\left(1+x^{2}\right)^{-\varrho / 2} \cdot: L^{2}\left(\mathbb{R}^{m}\right) \rightarrow H^{1}\left(\mathbb{R}^{m}\right)
\end{gathered}
$$

since $\partial_{j}, T: H^{1}\left(\mathbb{R}^{m}\right) \rightarrow L^{2}\left(\mathbb{R}^{m}\right)$ are bounded and the commutators $\partial_{j}-(1+$ $\left.x^{2}\right)^{\varrho / 2} \partial_{j}\left(1+x^{2}\right)^{-\varrho / 2}$. are obviously bounded on $L^{2}\left(\mathbb{R}^{m}\right)$, while the commutator $T-\left(1+x^{2}\right)^{\varrho / 2} T\left(1+x^{2}\right)^{-\varrho / 2}$. is bounded there by Lemma 3.2 .

By taking the Fourier transform, the uniform boundedness of (6.27) is equivalent to the estimates

$$
\left\|m\left(x_{n}, \xi\right) v\right\|_{H \varrho\left(\mathbb{R}^{m}\right)} \leq c(h, \varrho)\|v\|_{H \varrho\left(\mathbb{R}^{m}\right)}, \quad v \in C_{0}^{\infty}\left(\mathbb{R}^{m}\right), \quad x_{n} \in(0, h),
$$

where $m\left(x_{n}, \xi\right)=\exp \left(-x_{n} t(\xi)\right)$. Consider a decomposition $t=t^{(0)}+t^{(1)}$ as in (6.5), with $a=1, t^{(0)}=\chi t, t^{(1)}=(1-\chi) t$, and a cutoff function $\chi$ vanishing near $|\xi|=k$ so that $t^{(0)}$ is a smooth symbol. We introduce the multiplication operators $\mathcal{M}=$ $m\left(x_{n}, \xi\right) \cdot=\mathcal{M}^{(1)} \mathcal{M}^{(0)}, \mathcal{M}^{(j)}=\exp \left(-x_{n} t^{(j)}(\xi)\right) \cdot, x_{n} \in(0, h)$, and we check the uniform boundedness of

$$
\begin{array}{ll}
\mathcal{M}^{(0)}: H^{\varrho}\left(\mathbb{R}^{m}\right) \rightarrow H^{\varrho}\left(\mathbb{R}^{m}\right), & |\varrho| \leq 1, \\
\mathcal{M}^{(1)}: H^{\varrho}\left(\mathbb{R}^{m}\right) \rightarrow H^{\varrho}\left(\mathbb{R}^{m}\right), & |\varrho|<1 .
\end{array}
$$

Since we have, for $x_{n} \in(0, h)$ and $m_{0}\left(x_{n}, \xi\right)=\exp \left(-x_{n} t^{(0)}(\xi)\right)$,

$$
\left|m_{0}\left(x_{n}, \xi\right)\right| \leq c(h), \quad\left|\nabla_{\xi} m_{0}\left(x_{n}, \xi\right)\right| \leq c(h)\left(1+\xi^{2}\right)^{-1 / 2} \quad \text { on } \quad \mathbb{R}^{m},
$$

the norm of $(6.30)$ is bounded by some constant $c(h)$. To get a bound for $(6.31)$, we write

$$
\exp \left(-x_{n} t^{(1)}\right)=\sum_{j \geq 0} x_{n}^{j}\left(-t^{(1)}\right)^{j} / j !
$$

and apply the proof of Theorem 6.2 (ii) to estimate the norm of (6.31) by

$$
\sum_{j \geq 0} h^{j} c(\varrho)^{j} / j !=\exp (h c(\varrho)) .
$$

This finishes the proof of (6.27).

To prove the uniform boundedness of (6.28), we write

$$
\begin{aligned}
M_{0} & -\left(1+x^{2}\right)^{\varrho / 2} M_{0}\left(1+x^{2}\right)^{-\varrho / 2} \cdot=M^{(1)}\left(M^{(0)}-\left(1+x^{2}\right)^{\varrho / 2} M^{(0)}\left(1+x^{2}\right)^{-\varrho / 2} \cdot\right) \\
& +\left(M^{(1)}-\left(1+x^{2}\right)^{\varrho / 2} M^{(1)}\left(1+x^{2}\right)^{-\varrho / 2} \cdot\right)\left(1+x^{2}\right)^{\varrho / 2} M^{(0)}\left(1+x^{2}\right)^{-\varrho / 2} \cdot,
\end{aligned}
$$

where $M^{(j)}=F^{-1} \exp \left(-x_{n} t^{(j)}(\xi)\right) F, j=0,1$. By (6.32) and Remark 6.6 (iii), we obtain the uniform boundedness of

$$
M^{(0)}-\left(1+x^{2}\right)^{\varrho / 2} M^{(0)}\left(1+x^{2}\right)^{-\varrho / 2} \cdot: L^{2}\left(\mathbb{R}^{m}\right) \rightarrow H^{1}\left(\mathbb{R}^{m}\right) .
$$

Moreover, $M^{(1)}$ is obviously bounded on $H^{1}\left(\mathbb{R}^{m}\right)$ since its symbol is uniformly bounded. In view of (6.30) and the isometry $F: L_{\varrho}^{2}\left(\mathbb{R}^{m}\right) \rightarrow H^{\varrho}\left(\mathbb{R}^{m}\right)$, it is then 
sufficient to verify that the last commutator in (6.34) is uniformly bounded from $L^{2}\left(\mathbb{R}^{m}\right)$ into $H^{1}\left(\mathbb{R}^{m}\right)$, and for this it is enough to show the uniform boundedness (with respect to $x_{n}$ ) of

$$
N^{(1)}=M^{(1)}-I: L_{\varrho}^{2}\left(\mathbb{R}^{m}\right) \rightarrow H_{\varrho}^{1}\left(\mathbb{R}^{m}\right), \quad|\varrho|<1, \quad x_{n} \in(0, h),
$$

where $I$ is the identity operator and the symbol of $N^{(1)}$ is $n_{1}\left(x_{n}, \xi\right)=$ $\exp \left(-x_{n} t^{(1)}(\xi)\right)-1$. Taking the Fourier transform and using Remark 6.6 (ii), (6.35) is then equivalent to the uniform boundedness in $x_{n}$ of

$$
n_{1}\left(x_{n}, \xi\right) \cdot: H^{\varrho}\left(\mathbb{R}^{m}\right) \rightarrow H_{1}^{\varrho}\left(\mathbb{R}^{m}\right), \quad|\varrho|<1 .
$$

Consider the multiplication operator $\mathcal{N}^{(1)}=\left(1+\xi^{2}\right)^{1 / 2} n_{1}\left(x_{n}, \xi\right) \cdot$. Then, using relation (6.33), estimate (6.31) can be proved (in the same way) for $\mathcal{N}^{(1)}$ in place of $\mathcal{M}^{(1)}$; recall that $t^{(1)}$ has compact support. This easily implies (6.36), which finishes the proof of (6.28). Thus Lemma 3.4 (i) is proven.

\section{REFERENCES}

[1] H. Ammari, G. BaO, And A. W. Wood, Analysis of the electromagnetic scattering from a cavity, Japan J. Indust. Appl. Math., 19 (2002), pp. 301-310.

[2] T. Arens, S. N. Chandler-Wilde, and K. Haseloh, Solvability and spectral properties of integral equations on the real line: I. Weighted spaces of continuous functions, J. Math. Anal. Appl., 272 (2002), pp. 276-302.

[3] T. Arens, S. N. Chandler-Wilde, and K. Haseloh, Solvability and spectral properties of integral equations on the real line. II. $L^{p}$-spaces and applications, J. Integral Equations Appl., 15 (2003), pp. 1-35.

[4] T. ARENS AND T. Hohage, On radiation conditions for rough surface scattering problems, IMA J. Appl. Math., 70 (2005), pp. 839-847.

[5] G. Bao And D. C. Dobson, On the scattering by a biperiodic structure, Proc. Amer. Math. Soc., 128 (2000), pp. 2715-2723.

[6] A. S. Bonnet-Bendhia And P. Starling, Guided waves by electromagnetic gratings and nonuniqueness examples for the diffraction problem, Math. Methods Appl. Sci., 17 (1994), pp. 305-338.

[7] M. Cessenat, Mathematical Methods in Electromagnetism, World Scientific, Singapore, 1996.

[8] S. N. Chandler-Wilde, Boundary value problems for the Helmholtz equation in a half-plane, in Third International Conference on Mathematical and Numerical Aspects of Wave Propagation (Proceedings in Applied Mathematics), G. Cohen, ed., SIAM, Philadelphia, 1995, pp. $188-197$.

[9] S. N. Chandler-Wilde And B. Zhang, Electromagnetic scattering by an inhomogeneous conducting or dielectric layer on a perfectly conducting plate, Proc. R. Soc. Lond. Ser. A Math. Phys. Eng. Sci., A454 (1998), pp. 519-542.

[10] S. N. Chandler-Wilde And B. Zhang, A uniqueness result for scattering by infinite rough surfaces, SIAM J. Appl. Math., 58 (1998), pp. 1774-1790.

[11] S. N. Chandler-Wilde And P. Monk, Existence, uniqueness, and variational methods for scattering by unbounded rough surfaces, SIAM J. Math. Anal., 37 (2005), pp. 598-618.

[12] S. N. Chandler-Wilde And P. Monk, The PML for rough surface scattering, Appl. Numer. Math., 59 (2009), pp. 2131-2154.

[13] S. N. Chandler-Wilde, E. Heinemeyer, and R. Potthast, A well-posed integral equation formulation for three-dimensional rough surface scattering, Proc. R. Soc. Lond. Ser. A Math. Phys. Eng. Sci., 462 (2006), pp. 3683-3705.

[14] S. N. Chandler-Wilde, E. Heinemeyer, and R. Potthast, Acoustic scattering by mildly rough unbounded surfaces in three dimensions, SIAM J. Appl. Math., 66 (2006), pp. 10021026.

[15] S. N. Chandler-Wilde, P. Monk, And M. Thomas, The mathematics of scattering by unbounded, rough, inhomogeneous layers, J. Comput. Appl. Math., 204 (2007), pp. 549-559.

[16] S. N. Chandler-Wilde, M. Rahman, and C. R. Ross, A fast two-grid and finite section method for a class of integral equations on the real line with application to an acoustic scattering problem in the half-plane, Numer. Math., 93 (2002), pp. 1-51.

Copyright (c) by SIAM. Unauthorized reproduction of this article is prohibited. 
[17] S. N. Chandler-Wilde, C. R. Ross, and B. Zhang, Scattering by infinite one-dimensional rough surfaces, Proc. R. Soc. Lond. Ser. A Math. Phys. Eng. Sci., A455 (1999), pp. 37673787 .

[18] J. A. DeSanto, Scattering by rough surfaces, in Scattering and Inverse Scattering in Pure and Applied Science, R. Pike and P. Sabatier, eds., Academic Press, New York, 2002, pp. 15-36.

[19] T. M. Elfouhaily and C. A. GueRin, A critical survey of approximate scattering wave theories from random rough surfaces, Waves Random Complex Media, 14 (2004) pp. R1-R40.

[20] J. ElsChner And G. SCHMidt, Diffraction in periodic structures and optimal design of binary gratings I. Direct problems and gradient formulas, Math. Methods Appl. Sci., 21 (1998), pp. 1297-1342.

[21] J. Elschner, R. Hinder, F. Penzel, And G. Schmidt, Existence, uniqueness and regularity for solutions of the conical diffraction problem, Math. Models Methods Appl. Sci., 10 (2000), pp. 317-341.

[22] J. Elschner and M. Yamamoto, An inverse problem in periodic diffractive optics: Reconstruction of Lipschitz grating profiles, Appl. Anal., 81 (2002), pp. 1307-1328.

[23] K. O. HASELOH, Second kind integral equations on the real line: Solvability and numerical analysis in weighted spaces, Ph.D. thesis, Universität Hannover, Hannover, Germany, 2004.

[24] E. Heinemeyer, M. Lindner, And R. Potthast, Convergence and numerics of a multisection method for scattering by three-dimensional rough surfaces, SIAM J. Numer. Anal., 46 (2008), pp. 1780-1798.

[25] I. V. KAMOtSKil AND S. A. NAZAROV, Wood's anomalies and surface waves in the problem of scattering by a periodic boundary I, Sb. Math., 190 (1999), pp. 111-141.

[26] I. V. KamotskiǏ AND S. A. NAZAROV, Exponentially decreasing solutions of the problem of diffraction by a rigid periodic boundary, Math. Notes, 73 (2003), pp. 129-131.

[27] A. KIRsch, Diffraction by periodic structures, in Inverse Problems in Mathematical Physics, Proceedings of the Lapland Conference on Inverse Problems, L. Päivärinta and E. Somersalo, eds., Springer, Berlin, 1993, pp. 87-102.

[28] J.-L. Lions And E. Magenes, Problèmes aux Limites Non Homogènes et Applications, Dunod, Paris, 1968.

[29] A. Meier and S. N. Chandler-Wilde, On the stability and convergence of the finite section method for integral equation formulations of rough surface scattering, Math. Methods Appl. Sci., 24 (2001), pp. 209-232.

[30] A. MeIER, Numerical treatment of integral equations on the real line with application to acoustic scattering by unbounded rough surfaces, Ph.D. thesis, Brunel University, Uxbridge, UK, 2001.

[31] S. A. NAZARov, Artificial boundary conditions for finding surface waves in the problem of diffraction by a periodic boundary, Comput. Math. Math. Phys., 46 (2006), pp. 2164-2175.

[32] S. M. Nikol'SKIǏ, Approximation of Functions of Several Variables and Imbedding Theorems, Springer, New York, 1975.

[33] J. A. Ogilvy, Theory of Wave Scattering from Random Rough Surfaces, Adam Hilger, Bristol, UK, 1991.

[34] C. Parenti, Operatori pseudo-differenziali in $\mathbb{R}^{n}$ e applicazioni, Ann. Mat. Pura Appl., 93 (1972), pp. 359-389.

[35] M. Saillard and A. Sentenac, Rigorous solutions for electromagnetic scattering from rough surfaces, Waves Random Complex Media, 11 (2001), pp. R103-R137.

[36] M. A. Shubin, Almost periodic functions and partial differential operators, Russian Math. Surveys, 33 (1978), pp. 1-52.

[37] E. M. Stein, Singular Integrals and Differentiability Properties of Functions, Princeton University Press, Princeton, NJ, 1970.

[38] E. M. Stein and G. Weiss, Introduction to Fourier Analysis on Euclidean Spaces, Princeton University Press, Princeton, NJ, 1971.

[39] M. Thомas, Analysis of rough surface scattering problems, Ph.D. thesis, University of Reading, Reading, UK, 2006.

[40] H. Triebel, Interpolation Theory, Function Spaces, Differential Operators, North-Holland, Amsterdam, 1978

[41] L. R. Volevich and A. G. Mehtiev, Multipliers of Fourier integrals in weighted classes and imbedding theorems, Izv. Vyssh. Uchebn. Zaved. Mat., 65 (1967), pp. 27-38 (in Russian).

[42] A. G. Voronovich, Wave Scattering from Rough Surfaces, Springer, Berlin, 1994.

[43] K. F. WARNICK AND W. C. ChEW, Numerical simulation methods for rough surface scattering, Waves Random Complex Media, 11 (2001), pp. R1-R30.

Copyright $@$ by SIAM. Unauthorized reproduction of this article is prohibited. 